\title{
Ontologia para Configuração Semi-Automática de Redes de Valor
}

\section{Ontology for Semi-Automatic Configuration of Value Networks}

\author{
Jefferson da Silva Reis, Patrício de Alencar Silva e Angélica Félix de Castro \\ Programa de Pós-Graduação em Ciência da Computação - Universidade Federal Rural \\ do Semi-Árido (UFERSA) \\ Mossoró, RN - Brasil \\ sreis.jefferson@gmail.com, \{patricio.alencar, \\ angelica.castro\}@ufersa.edu.br
}

\begin{abstract}
This paper describes an ontology for leveraging value networks modeling. Currently, this modeling technique demands substantial tacit knowledge from a business analyst, which could be represented in a machinereasonable language to enable knowledge management in an agile enterprise. The ontology is based on concepts of Multiple Agency Theory, Enterprise Ontology, Value Network Modeling and Speech Acts Theory, supporting semiautomatic configuration of value network models. The utility of the ontology is evaluated by means of three scenarios of real-world case studies. Case analysis was based on an observational case study protocol adapted from Design Science.
\end{abstract}

Keywords. Design Science, Enterprise Ontology, Value Network Modeling.

Resumo. Este artigo descreve uma ontologia para otimizar a modelagem de redes de valor. Este tipo de modelagem ainda depende consideravelmente do conhecimento tácito do analista de negócios, o qual pode ser representado em linguagem de máquina para facilitar a gestão do conhecimento em uma empresa ágil. A ontologia proposta é baseada em conceitos de Teoria de Agência Múltipla, Enterprise Ontology, Modelagem de Redes de Valor e Teoria dos Atos de Fala, permitindo a configuração semiautomática de modelos de redes de valor. A utilidade da ontologia é avaliada com três cenários de estudo de casos reais. A análise dos casos foi baseada em um protocolo de estudo de caso observacional adaptado de Design Science.

Palavras-Chave. Design Science, Enterprise Ontology, Modelagem de Redes de Valor.

\section{Introdução}

Uma rede de valor foi originalmente referida como uma rede de empresas permutando objetos de valor econômico para satisfazer as necessidades de um mercado de consumidores [Normann e Ramirez 1993]. Deste modo, as redes de valor representam arranjos de atores, atividades e objetos de valor econômico que configuram modelos de negócio. Estes arranjos organizacionais formam um contrato de cooperação que antecede a exploração de um determinado segmento de mercado [de Alencar Silva et al. 2017]. No

Cite as: $\quad$ Reis, J. S., Silva, P. A.. \& Castro, A. F. (2020). Ontology for Semi-Automatic Configuration of Value Networks (Ontologia para Configuração Semi-Automática de Redes de Valor). iSys: Revista Brasileira de Sistemas de Informação (Brazilian Journal of Information Systems), 13(1), 77-113. 
entanto, a modelagem de rede de valor demanda um conhecimento tácito considerável dos analistas de negócio. Para dar suporte a essa tarefa, foram surgindo ferramentas de suporte à modelagem de redes de valor.

Com o objetivo de definir formalmente como os atores empresariais poderiam colaborar dentro de uma rede de valor, Gordijn e Akkermans (2003) propuseram um framework, denominado esvalue, para modelagem e análise de redes de valor. Esse framework é baseado em uma ontologia semiformal, descrita em Unified Modeling Language (UML). O pilar dessa ontologia é a noção de sustentabilidade econômica, que define como os atores criam, trocam e consomem objetos de valor econômico. A sustentabilidade econômica é vital para o funcionamento de uma rede de valor, pode deve ser rentável para todos os atores envolvidos. O esvalue é uma abordagem de modelagem que é originalmente destinada a apoiar a exploração de novas redes de negócios. Nesta fase, a coordenação temporal das transações de valor são irrelevantes. $\mathrm{O}$ aspecto mais importante a ser analisado nesta fase é possibilidade que o modelo oferece de criação de valor positivo para todos os participantes [Weigand et al. 2007].

O princípio fundamental que rege a ontologia do esvalue é o de reciprocidade econômica, que define como os atores empresariais sacrificam objetos de valor econômico para obter outros (de valor equivalente) em troca. Esse modelo de negócio leva em consideração apenas valores monetários em uma transação de valor. Atingir os objetivos estabelecidos para esse valor objetivo é necessário para a sustentabilidade econômica de uma rede de valor, mas não é suficiente para um consumidor declarar que sua empresa precisa estar plenamente satisfeita com tal medida de valor [de Alencar Silva et al. 2017] [Weigand et al. 2007]. Portanto, valores subjetivos tais como garantia, privacidade e confiança, podem ser relevantes para que um consumidor não somente diferencie propostas de valor monetário equivalentes, mas também para adquirir produtos ou serviços cuja avaliação depende da experiência ou uso, tais como os serviços vendidos pelo comércio eletrônico (e.g. pacotes de turismo) ou produtos inovadores (p. ex. gadgets).

O foco desta pesquisa está na especificação de um modelo semântico para configuração semiautomática de modelos de redes de valor. Atualmente, a representação desses modelos é limitada a modelos estáticos em UML complementados por regras descritas em Object Constraint Language (OCL). Por exemplo, apesar de prover um mecanismo robusto de cálculo de rentabilidade, a ferramenta esvalue limita-se a uma ferramenta desktop que dificulta a interoperabilidade na comunicação de modelos de redes de valor. Em vista a complexidade crescente das redes de valor pela grande quantidade de relações entre atores envolvidos nos negócios (i.e., fornecedores, terceiros, órgãos governamentais e consumidores), torna-se necessária a construção de novas tecnologias de suporte à configuração ágil dessas redes, facilitando assim a comunicação entre as partes interessadas. Sendo assim, faz-se necessária a especificação de um modelo descrito em linguagem interpretável por máquina que defina a organização interna dos elementos de um modelo de rede de valor. Adotando uma perspectiva de pesquisa em Design Science conforme estruturada por Wieringa (2014a), a questão de pesquisa principal deste trabalho é como um modelo de rede de valor pode ser configurado de forma semiautomática. Do ponto de vista organizacional [Cameron 1980], esta questão demanda investigação sobre o levantamento de requisitos de construção de um metamodelo para especificação formal de modelos de redes de valor e avaliação da utilidade potencial deste meta-modelo. 
Para tratamento dessas questões de pesquisa, propomos uma ontologia para configuração semiautomática de modelos redes de valor. A ontologia foi especificada em OWL-DL 2 (Web Ontology Language 2 fundamentada em description logic - DL para representação do conhecimento) e complementada por um conjunto de regras definidas em Semantic Web Rule Language (SWRL) [Horrocks et al. 2004], combinando conceitos da ontologia de base do esvalue [Gordijn 2002] com conceitos de organização de modelos formais de estratégias e processos de negócios adaptados de Enterprise Ontology [Dietz 2006], Speech Acts [Searle e Vanderveken 1985] e Value Monitoring Ontology (VMO) [de Alencar Silva e Weigand 2011].

Uma rede de valor semântica não é uma nova rede de valor, ou seja, não está sendo discutido um novo conceito para redes de valor. Entretanto, uma rede de valor semântica corresponde a uma rede de valor construída com auxílio de tecnologias da web semântica. Uma rede de valor semântica pode apresentar visões diferentes, possibilitar reconfigurações de modelos, auxiliar na modelagem da rede de valor e inferir conhecimento a partir da rede. Em outros termos, pretende-se aqui utilizar serviços da web semântica para representação do domínio das redes de valor.

A relevância deste trabalho está na ausência de uma ontologia formal para modelagem de redes de valor, utilizando-a como um artefato para os Sistemas de Informação. A modelagem de redes de valor é uma tarefa que consome tempo e recursos humanos que poderiam ser melhor utilizados. Além disso, a representação do domínio de redes de valor propostas por trabalhos relacionados a esta pesquisa, está normalmente baseada em modelos semi-formais enriquecidos com padrões de modelagem que podem ser aplicados na modelagem das redes de valor. No entanto, não abordam melhores práticas para representação do conhecimento seguida de estratégias que possam auxiliar analistas de negócios a desenvolverem modelos de redes de valor. Sendo assim, a direção a ser tomada nesta pesquisa é apresentar uma ontologia formal como estratégia de modelagem de redes de valor, que poderá posteriormente ser configurada como parte de um Sistema de Informação a ser implementado numa plataforma de apoio à decisão.

O restante deste artigo está organizado como se segue. Na Seção 2, é apresentado um referencial teórico sobre os conceitos usados nesta pesquisa, com ênfase em modelagem de redes de valor, Enterprise Ontology e os Teoria dos Atos de Fala. Na Seção 3, é apresentada a especificação formal da ontologia de modelagem de redes de valor semânticas. A Seção 4 reporta sobre a análise dos cenários de estudos de caso usados para avaliar a utilidade potencial da ontologia, de acordo com as diretrizes de um protocolo de estudo de caso observacional adaptado da literatura de Design Science. A Seção 5 descreve os trabalhos relacionados a esta pesquisa e a Seção 6 apresenta os resultados preliminares e direções para pesquisas futuras.

\section{Fundamentação Teórica}

Uma ontologia formal fornece uma semântica para o raciocínio automático em redes de valor, a fim de facilitar a modelagem, análise e a verificação das redes de valor. As ontologias fornecem uma representação explícita e formal da rede de valor. Além do mais, são utilizadas em vários campos, tais como: para extração de informações, gestão de conhecimento e na web semântica. Possibilitando a comunicação entre atores, processos, sistemas, dentre outros elementos, que fazem parte de um mesmo domínio do conhecimento. 
As ontologias podem ser classificadas segundo Guarino et al. (1998), de acordo com seu grau de generalização: genérica, tarefa, domínio e aplicação. De acordo com Morais e Ambrósio (2007), as ontologias genéricas são consideradas gerais por descreverem conceitos amplos (p.ex. natureza, espaço, tempos e outros). Já ontologias de domínio apresentam conceitos e vocabulários relacionados a domínios particulares. A ontologia apresentada neste trabalho corresponde a uma ontologia de tarefa, essas ontologias descrevem tarefas ou atividades genéricas que podem contribuir para a resolução de um problema. Por fim, ontologias de aplicação apresentam conceitos que definem atividades específicas, como uma especialização dos termos específicos das ontologias de domínio e de tarefas. As ontologias tem sido uma área de pesquisa abrangente, com diversas formas de representá-las, sendo a Web Ontology Language (OWL-DL) o padrão adotado pelo World Wide Web Consortium (W3C).

Modelos de redes de valor esboçam contratos multilaterais de cooperação entre atores de negócios para exploração de um segmento de mercado. Algumas teorias têm sido propostas para explicar como se deve estruturar uma rede de valor. Dentre as principais teorias propostas, o ezvalue consiste de um framework para análise e uma ontologia para modelagem de redes de valor [Gordijn e Akkermans 2007]. O foco desta abordagem é prever a sustentabilidade econômica de uma rede de valor [Weigand et al. 2007]. Para mitigar riscos de oportunismo de atores participantes de uma rede de valor, de Alencar Silva e Weigand (2011) estenderam o framework esvalue com requisitos de monitoramento preventivo de atividades de valor suspeitas com a VMO. O objetivo da VMO é prover suporte à especificação de modelos de redes de valor robustos contra fraudes por omissão de informação sobre a real capacidade de um ator de entregar, em tempo de realização do negócio, o produto ou serviço prometido em tempo de acordo.

A Enterprise Ontology é outra abordagem para modelagem de negócios proposta por Dietz (2006). Segundo o autor, as organizações são essencialmente sistemas sociais, cujos elementos são seres humanos, que no seu papel de sujeitos sociais, possuem autoridade e responsabilidade para a execução de atos. Esta abordagem explora a visão das ações internas de uma empresa, descrevendo seus processos constituintes com padrões de comunicação adaptados da Teoria de Atos de Fala, proposta no campo da linguística por Searle (1969). De acordo com esta última teoria, agentes internos de uma empresa se comunicam por atos de produção (i.e. production acts ou p-acts; e.g. produção, utilização ou consumo) e atos de coordenação (i.e., coordination acts ou $c$ acts; e.g. oferta ou aceitação). Uma operação de produção liga um agente a um recurso da empresa., enquanto que uma operação de coordenação liga um agente a outro agente. O axioma transacional da teoria define um padrão de comunicação entre atos de coordenação, o qual pode envolver dois atores com objetivo de alcançarem um determinado resultado. O axioma de composição especifica transações elementares podem ser compostas na formação de processos de negócio.

A Teoria de Atos de Fala proposta por Searle (1969) no campo de estudo da Linguística Aplicada inspirou o desenvolvimento de muitas aplicações na Computação, especialmente na especificação de protocolos de comunicação multiagente, em que agentes de software racionais atribuem significado às suas ações e planos de execução correspondentes. A Teoria dos Atos de Fala pode ser adaptada para definir perfis de comportamento através da comunicação. Por exemplo, a classificação de atos de fala proposta por Searle e Vanderveken (1985) pode ser combinada com o model Role-Based Acccess Control (RBAC) [Ferraiolo et al. 2001] - ou Modelo de Controle de Acesso 
baseado em Responsabilidades) em tradução livre - para classificar o comportamento de agentes empresariais.

Para especificar a ontologia de modelagem de redes de valor semânticas, combinamos conceitos centrais das teorias descritas acima, os quais julgamos essenciais para a representação formal e organização dos elementos componentes da rede. A próxima seção descreve a especificação formal da ontologia

\section{Ontologia de Rede de Valor Semântica}

A ontologia proposta 1 foi construída com o objetivo de fornecer uma especificação formal dos principais elementos que compõem um modelo de rede de valor. A ontologia descrita em OWL2 e complementada com regras descritas em SWRL permite a configuração semiautomática de modelos de redes de valor e inferência semiautomática de conhecimento sobre a previsão de geração de valor subjetivo. A ontologia é composta por 43 classes e 62 propriedades de objetos, que podem ser resumidas em três níveis distintos de abstração, conforme ilustrado na Figura 1. O primeiro nível contém as relações entre os atores, atividades e objetos de valor que constituem a base de qualquer rede de valor. No segundo nível, encontram-se os valores objetivos e subjetivos dos produtos ou serviços da rede. No terceiro nível, estão as políticas organizacionais e a necessidade de negócio do consumidor.

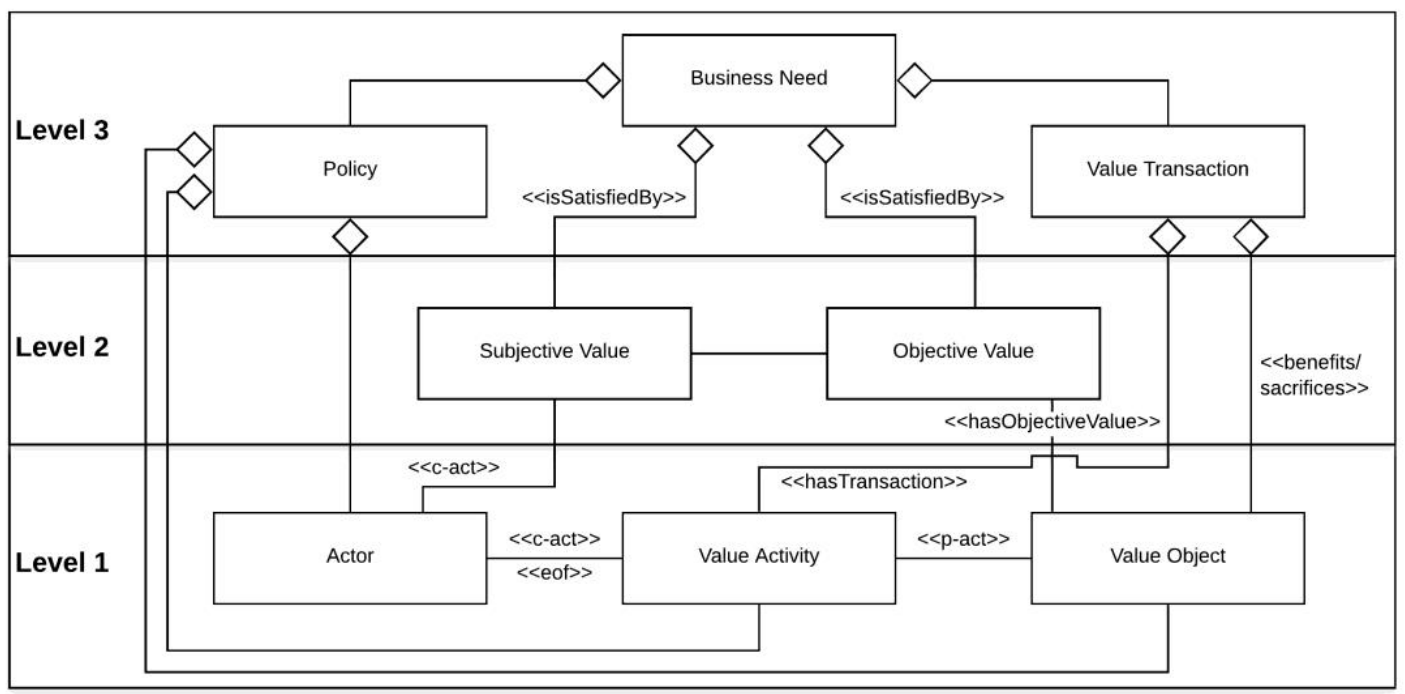

Figura 1. Representação da ontologia em UML

O desenvolvimento da ontologia seguiu os princípios da engenharia ontológica que se refere ao conjunto de atividades que norteiam todo o processo de desenvolvimento da ontologia [Gomez-Perez et al. 2006]. A disciplina de Engenharia Ontológica investiga princípios, métodos, metodologias, ferramentas e linguagens que dão suporte no ciclo de vida da ontologia [Gomez-Perez et al. 2006; Suer et al. 2009]. As metodologias propostas na literatura auxiliam no desenvolvimento ontológico, visando a qualidade no processo de especificação da ontologia. A construção da ontologia seguiu a Metodologia de Engenharia de Ontologias proposta por Sure, Staab e Studer (2009), que considera seis 
fases no processo de construção da ontologia, a saber: estudo de viabilidade, kickoff, refinamento, avaliação, aplicação e evolução.

Na primeira etapa, têm-se o estudo de viabilidade, em que, pode-se identificar problemas de pesquisa, oportunidades de pesquisa e potencias soluções que justificam a construção da ontologia. Como a falta de uma semântica formal para modelagem de redes de valor, impossibilita a inferência sobre os elementos da rede, tornando o seu processo de desenvolvimento uma atividade totalmente manual [de Alencar Silva et al., 2017]. Uma ontologia pode ser utilizada como parte da solução deste problema. Então, o objetivo é especificar uma ontologia formal capaz de permitir a busca, a instanciação e seleção de modelos, auxiliando analistas de negócios e engenheiros de software a desenvolver suas redes de valor. Foram identificadas as ferramentas e tecnologias necessárias para a especificação da ontologia, dentre elas: Web Ontology Language (OWL), a Semantic Web Rule Language (SWRL) e o editor de ontologias de código aberto Protégé.

Seguindo para segunda etapa do processo de desenvolvimento, foi necessário capturar os requisitos e criar uma descrição semiformal da ontologia. A especificação dos requisitos do software é um processo chave no desenvolvimento de software por definir as funcionalidades necessárias acerca do componente de software [Sommerville 2011]. Nesta etapa é elaborado o documento de especificação de requisitos da ontologia, que tem o objetivo de definir o propósito da ontologia, o seu uso pretendido, quem são seus usuários finais e quais os requisitos que a ontologia deve cumprir [Suárez-Figueroa et al. 2009]. Como parte dos requisitos, foram definidas algumas questões de competência que a ontologia após instanciada seja capaz de responder, podendo assim determinar o alcance da ontologia [Grüninger and Fox 1995]. Ainda nesta etapa, pode-se determinar o domínio e o escopo da ontologia [Noy and Mcguinness 2001]. A Tabela 1, apresenta o documento de especificação de requisitos elaborado para Ontologia de Redes de Valor Semânticas. Este documento segue as orientações metodológicas propostas por Suárez-Figueroa, Suárez-Figueroa et al. (2009). O resultado da atividade de especificação é um modelo com informações sobre o objetivo, o escopo, a linguagem de implementação, os usuários finais previstos, casos de uso, requisitos funcionais, requisitos não funcionais e o préglossário de termos da ontologia.

Tabela 1. Documento de Requisitos da Ontologia

\begin{tabular}{|l|l|}
\hline \multicolumn{3}{|c|}{ DOCUMENTO DE ESPECIFICAÇÃO DE REQUISITOS DA SVNO } \\
\hline $\mathbf{1}$ & Objetivo \\
\hline $\begin{array}{l}\text { O objetivo da construção da Ontologia de Redes de Valor Semânticas é fornecer um } \\
\text { modelo semântico capaz de buscar, instanciar, selecionar e gerar modelos de forma } \\
\text { semiautomática que auxilie analistas de negócios a modelar redes de valor, além de } \\
\text { fornecer um conhecimento consensual das relações entre os elementos dos modelos } \\
\text { de valor. }\end{array}$ \\
\hline $\mathbf{2}$ & Escopo \\
\hline $\begin{array}{l}\text { A ontologia define o domínio das Redes de Valor. O nível de granularidade está } \\
\text { diretamente relacionado às questões de competência e aos termos identificados. Além } \\
\text { disso, por especificar um vocabulários próprio de um domínio especifico, deve ser } \\
\text { classificada como uma ontologia de domínio. }\end{array}$ \\
\hline $\mathbf{3}$ & Linguagem de Implementação \\
\hline
\end{tabular}

iSys: Revista Brasileira de Sistemas de Informação (iSys: Brazilian Journal of Information Systems) http://seer.unirio.br/index.php/isys/ 
A ontologia deve ser especificada em OWL-DL 2 (Web Ontology Language Descripton Logic 2 version).

4 Usuários Finais Previstos

1 - Gestores e empreendedores que desejam elaborar, estratégias de redes de valor; 2 Organizações públicas ou privadas que desejam analisar acordos de gestão estruturados em modelos de negócio.

5 Casos de Uso

Auxiliar na especificação de redes de valor; Armazenar, buscar e atualizar redes de valor; Fornecer análise qualitativa das redes de valor.

6 Requisitos da Ontologia

\section{a. Requisitos Não-Funcionais}

A ontologia deve prover suporte a cenários multilíngues, em pelo menos dois idiomas (p.ex. Português e Inglês); A terminologia utilizada na ontologia deve ser retirada do domínio do estado da arte das redes de valor seguindo as questões conceituais apresentadas nas questões de pesquisa; A ontologia deve ser baseada no framework esvalue, na Value Monitoring Ontology e na Enterprise Ontology.

\section{b. Requisitos Funcionais: Grupo de Questões de Competência}

QC1: Quais são as responsabilidades dos atores de uma rede de valor?

QC2: Quais são os tipos de atividades realizadas pelos atores da rede?

QC3: Quais são os objetos de valor comunicados entre os atores?

QC4: Como recuperar políticas organizacionais que compõem uma rede de valor?

QC5: Como verificar se transações da rede são economicamente sustentáveis (reciprocidade econômica)?

QC6: Como os valores subjetivos e objetivos podem garantir a satisfação do ator em relação a sua necessidade de negócio?

7 Pré-Glosário de Termos

\section{a. Termos de Questões de Competência + Frequência}

Ator + 07; Valor Subjetivo + 03; Objetos de Valor + 02; Atividades + 01; Valor Objetivo + 01; Valor Subjetivo + 1; Políticas +1 ; Transações +1 ; Necessidade de Negócio + 1;

\section{b. Objetos}

Surplus, Balance, Shortage; Opportunity, Threat, Weakness. Deserved Value, Equitable Value, ForecastedValue, Ideal Value, Minimum Tolerable Value.

Para formalizar uma ontologia alvo, foram necessários ciclos de refinamento. Alguns aspectos importantes sobre modelagem de ontologias foram considerados nestas etapas: (1) identificação da necessidade de se utilizar partições de valores; (2) definição de classes disjuntas; (3) criação de axiomas de fechamento; (4) concatenação de 
propriedades; (5) uso da linguagem de regras da web semântica (SWRL); (6) definição de propriedades inversas; (7) documentação das classes e propriedades da ontologia; (8) e o refinamento dos conceitos da ontologia alvo. A ontologia proposta fornece a classificação de todos os elementos de uma rede de valor. Por meio de uma descrição formal, descreve como os elementos podem ser combinados, ou seja, permite que modelos de negócios possam ser instanciados em um modelo semântico.

A seguir, são apresentados detalhes da formalização das classes da ontologia descrita em sintaxe Manchester para OWL [Horridge et al. 2006]. O ponto de partida é a definição axiomática das classes que estão no primeiro nível de abstração da ontologia. A sintaxe Manchester descreve as estruturas de lógica de descrição baseada em frames. Para definir uma classe utiliza-se a palavra "Class" que indica o nome da classe. Em seguida, "EquivalentTo" apresenta as relações axiomáticas para classes definidas, enquanto que classes primitivas são representas por "SubClassOf".

\subsection{Definindo atores, atividades de valor e objetos de valor}

Uma rede de valor é composta principalmente de atores, atividades e objetos de valor econômico. Um ator é definido como uma entidade juridicamente independente (empresa ou organização) que possui uma responsabilidade econômica de mercado [Gordijn 2014]. Em uma rede de valor os atores podem ser identificados pelo papel de agência que desempenham [Eisenhardt 1989]. Assim como abordado por de Alencar Silva (2013), quatro papéis distintos podem ser atribuídos aos atores em uma relação de agência: principal, agente, terceiro ou regulador. Segundo a Enterprise Ontology, os atores podem ser ligados a atos de produção (equivalentes atividades de valor em esvalue) por relações de competência, responsabilidade ou autoridade [Dietz 2006]. Além disso, atos de coordenação podem ligar atores a outros elementos da rede, assim como apresentado na Listagem 1 abaixo.

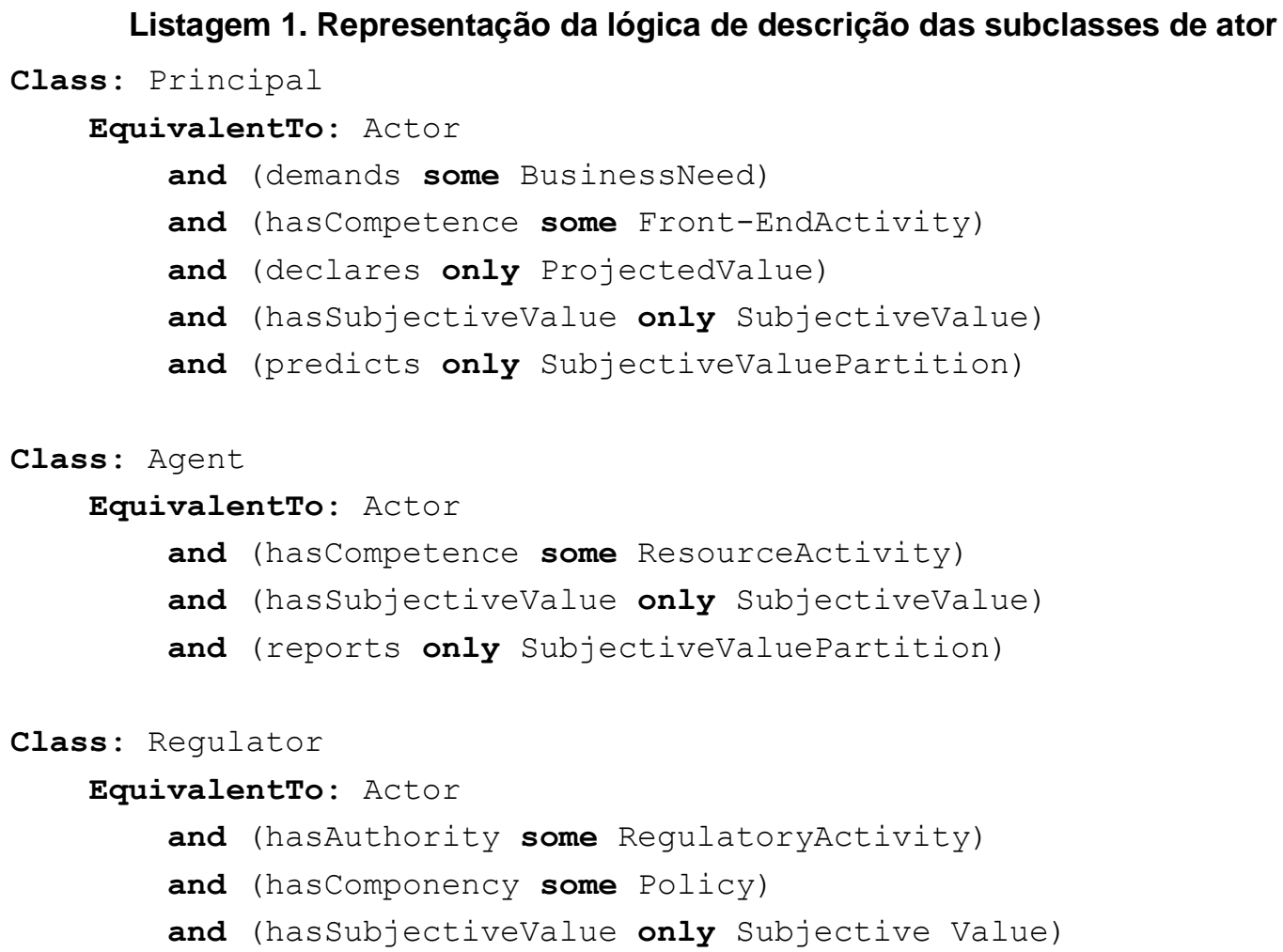

Listagem 1. Representação da lógica de descrição das subclasses de ator 
and (testifies only subjectiveValuePartition)

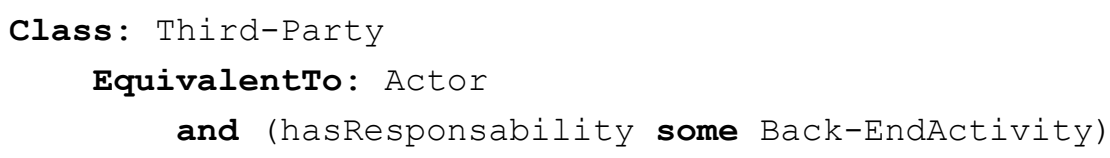

Os atores, as atividades e os objetos de valor são mutuamente definidos por forças de autoridade, competência e responsabilidade (ator-atividade) e atos de produção (atividade-objeto) que definem tarefas de transformação dos objetos de valor (e.g. consumir, produzir, distribuir, conceder, agregar ou transferir). Uma rede de valor pode ter quatro tipos de atividades: front-end, back-end, atividade-recurso e atividade regulatória. Cada ator realiza um tipo de atividade específica. Quando uma atividade que é realizada por mais de um ator do mesmo tipo, o conjunto de atores passa a ser um segmento de mercado. As descrições axiomáticas das atividades baseiam-se na interação destas com os objetos de valor. Portanto, uma atividade pode ter um benefício econômico (i.e., consumir ou agregar um objeto de valor) e em troca deve sacrificar um objeto de valor econômico correspondente (i.e., distribuir, produzir, transferir ou conceder um objeto de valor), conforme ilustrado na Listagem 2 a seguir.

\section{Listagem 2. Lógica de descrição dos tipos de atividade de valor}

class: Front-EndActivity

EquivalentTo: ValueActivity

and ( (bundles some

(CnAObject or PoPObject)) or

(consumes some Coreobject))

and (produces some Counterobject)

and (iscompetenceof some Principal)

and (hastransaction some ValueTransaction)

Class: ResourceActivity

EquivalentTo: ValueActivity

and ( (bundles some

(CnAobject or Coreobject or

Counterobject or PoPObject)) or

(consumes some Counterobject))

and ( distribute some

(Coreobject or Counterobject)) or

(grants some PoPObject) or

(transfers some CnAobject))

and (iscompetenceOf some Agent)

and (hastransaction some ValueTransaction)

Class: RegulatoryActivity

EquivalentTo: ValueActivity

and ( (bundles some

(CnAObject or CoreObject or PoPObject)) or

(consumes some Counterobject))

and ( (grants some CnAObject) or 

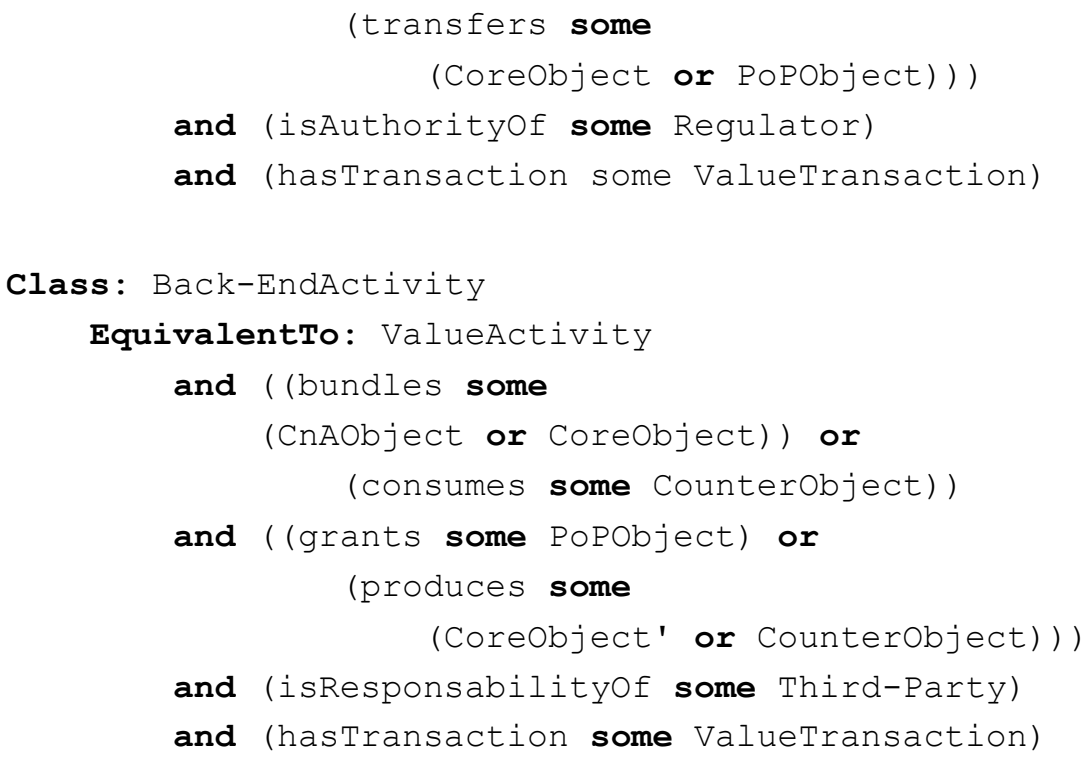

Toda atividade realiza pelo menos uma transação de valor, onde o sacrifício indica que a atividade envia um objeto de valor para a rede. Para cada sacrifício, deve existir um benefício correspondente indicando que atividade recebeu um pagamento em troca. As regras de reciprocidade econômica definem o que é benefício ou sacrifício para os agentes da rede. Assim, se uma atividade de valor agrega ou consome um objeto de valor, então tem como benefício esse objeto. Entretanto, se a atividade de valor produz, transfere, concede ou distribui, tem como sacrifício o objeto sobre o qual incidem essas operações. Um exemplo é apresentado na Tabela 2 abaixo, no qual a atividade front-end e back-end permutam um objeto principal de negócio. Esta lógica permite a análise das transações de valor descritas posteriormente neste artigo, associando o benefício e o sacrifício de cada atividade a seu respectivo objeto de valor.

Tabela 2. Regras SWRL para atribuição de benefícios e sacrifícios

\begin{tabular}{|c|c|}
\hline $\begin{array}{l}\text { Definição } \\
\text { de Valores }\end{array}$ & Regras SWRL para atribuição do benefício ou sacrifício \\
\hline Benefício & $\begin{array}{l}\text { Front-EndActivity(?atv) ^ Coreobject(?obj) } \\
\text { consumes(?atv, ?obj) -> hasBenefit(?at1, ?obj1) }\end{array}$ \\
\hline Sacrifício & $\begin{array}{l}\text { Back-EndActivity (?atv) ^ Coreobject (?obj) } \\
\text { produces(?atv, ?obj) } \rightarrow \text { hasSacrifice (?at1, ?obj1) }\end{array}$ \\
\hline
\end{tabular}

Por serem mutuamente definidos, os objetos de valor são definidos por relações com as atividades de valor. Um objeto de valor é um produto, serviço ou experiência de valor econômico para pelo menos um dos atores envolvidos [Gordijn e Akkermans 2003]. Segundo Dietz (2006), os objetos são bens ou serviços adquiridos como resultados de uma atividade. Na ontologia a classe objetos de valor possui quatro subclasses distintas: core object (i.e. objeto principal de negócio), proof-of-performance object (prova ou evidência de entrega de produto ou serviço), certification-and-accreditation object (i.e. certificação e acreditação) e counter-object (contra-objeto). A Listagem 3 descreve cada objeto com propriedades inversas para interação com as atividades de valor.

\section{Listagem 3. Lógica de descrição dos tipos de objeto de valor}

Class: Coreobject

EquivalentTo: Valueobject 
and ( ( isBundledBy some

(Back-EndActivity or ResourceActivity)) or

(isconsumedBy some Front-EndActivity))

and ((isDistributedBy some Resource Activity) or

(isProducedBy some Back-EndActivity))

Class: Counterobject

Equivalentтo: Valueobject

and ((isBundledBy some ResourceActivity)

or (isconsumedBy some

(Back-EndActivity or RegulatoryActivity

or ResourceActivity)) )

and ((isDistributedBy some ResourceActivity)

or (isproducedBy some

(Back-End Activity' or Front-EndActivity)))

Class: PoPObject

EquivalentTo: Valueobject

and (isBundledBy some

(Front-EndActivity or RegulatoryActivity

or ResourceActivity))

and (isGrantedBy some

(Back-EndActivity or ResourceActivity))

Class: CnAObject

EquivalentTo: Valueobject

and ((isGrantedBy some RegulatoryActivity) or

(isTransferredBy some ResourceActivity))

and (isBundledBy some

(Back-EndActivity or Front-EndActivity

or ResourceActivity))

\subsection{Definindo valores objetivos e valores subjetivos}

Uma proposição de valor é uma promessa de um ator para satisfazer a necessidade de um consumidor. Essa promessa de valor é formada por objetos e indicadores de valor. A proposição de valor de uma empresa não está somente ligada aos seus objetos de valor, mas principalmente a seus indicadores de valor. Por apresentarem valores tangíveis aos objetos de valor, os valores objetivos representam características que auxiliam no processo de decisão sobre qual objeto de valor é mais adequado para satisfazer a necessidade de negócio do consumidor. A propriedade isobjectivevalue liga um valor objetivo ao seu respectivo objeto de valor. Valores objetivos incluem localização, qualidade, quantidade ou tempo de permuta de um objeto (cf. Listagem 4). As subclasses podem ser enriquecidas com ontologias de alto nível, como por exemplo, a OWL-Time [Hobbs e Pan 2006] que descreve conceitos e propriedades temporais, a ontologia Mathematics [Gruber and Olsen 1994] para expressar quantidades ou a ontologia de O'Sullivan (2006) que descreve parâmetros de qualidade de serviço. 


\section{Listagem 4. Lógica de descrição de indicadores de valor objetivo}

Class: ObjectiveValue

EquivalentTo: ValueIndicator and

(Location or Quality or

Quantity or Time)

Subclassof: isobjectiveValue some Valueobject

Diferentes dos valores objetivos, os valores subjetivos são utilizados pelos consumidores para avaliar um produto ou serviço antes da aquisição. Sem experiência no uso do produto ou serviço, é comum que os consumidores considerem a avaliação de outros atores sobre a mercadoria desejada. Tal prática não é recente e, portanto, não deve estar exclusivamente associada à atual tendência de soluções de comércio eletrônico. Um valor subjetivo tem duas funções, cuja definição depende de quem comunica a avaliação [Biggemann e Buttle 2012], podendo estes serem expressos como valor ideal ou valor experimentado (vide Listagem 5). As expectativas (ou seja, o valor ideal) são vistas como previsões feitas pelos consumidores sobre o valor a ser retornado pelo objeto ou serviço [Parasuraman et al. 1988]. O valor experimentado é a avaliação geral dos consumidores com base nas percepções sobre o que é recebido [Zeithaml 1988]. O valor experimentado é um dos fatores que mais influenciam a satisfação do consumidor [Parasuraman et al. 1988].

Os valores subjetivos são aferidos por meio de uma escala de cinco níveis de valoração extraídos do modelo SERVQUAL. A escala define que a aferição de valor pode ser ideal, previsto, equitativo, merecido ou minimamente tolerável [Parasuraman et al. 1988]. Dessa forma, para que o principal declare que um objeto de valor satisfaz sua necessidade de negócio, este depende da avaliação de agentes ou reguladores que já experimentaram o serviço ou usaram o produto. A Tabela 3 sumariza as regras SWRL para definição do valor projetado com base no confronto entre valor ideal e valor experimentado.

Tabela 3. Regras SWRL para atribuição de valor projetado

\begin{tabular}{|c|c|}
\hline $\begin{array}{l}\text { Partição de } \\
\text { Valor } \\
\text { Projetado }\end{array}$ & Regras SWRL para atribuição de valor projetado \\
\hline $\begin{array}{c}\text { Value } \\
\text { surplus }\end{array}$ & $\begin{array}{c}\text { Principal (?p)`demands (?p, ?bn)^ } \\
\text { BusinessNeed (?bn)^hasSubjectiveValue (?p, } \\
\text { ?sv)`hasIdealValue (?sv, EquitableValue)^ } \\
\text { hasExperiencedValue (?sv, IdealValue) } \\
\rightarrow \text { hasProjectedValue (?bn, Surplus) }\end{array}$ \\
\hline $\begin{array}{c}\text { Value } \\
\text { balance }\end{array}$ & $\begin{array}{c}\text { Principal (?p)^demands (?p, ?bn)^ } \\
\text { BusinessNeed (?bn)^hasSubjectiveValue (?p, } \\
\text { ?sv)`hasIdealValue (?sv, } \\
\text { EquitableValue) hasExperiencedValue (?sv, EquitableValue) } \\
\rightarrow \text { hasProjectedValue (?bn, balance) }\end{array}$ \\
\hline $\begin{array}{c}\text { Value } \\
\text { shortage }\end{array}$ & $\begin{array}{c}\text { Principal (?p)^demands (?p, ?bn)^ } \\
\text { BusinessNeed (?bn)^hasSubjectiveValue (?p, } \\
\text { ?sv)`hasIdealValue (?sv, } \\
\text { EquitableValue)`hasExperiencedValue(?sv, DeservedValue) } \\
\rightarrow \text { hasProjectedValue(?bn, shortage) }\end{array}$ \\
\hline
\end{tabular}




\section{Listagem 5. Lógica de descrição de indicadores de valor subjetivo}

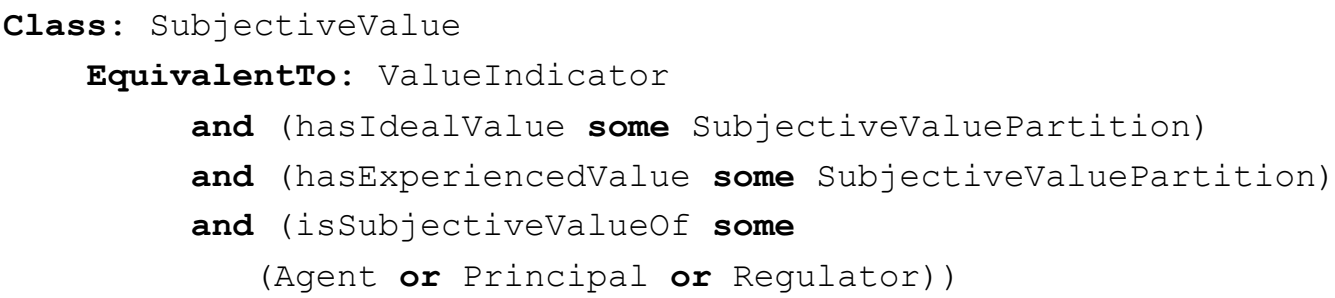

\subsection{Definindo políticas, transações e necessidades de negócios}

O terceiro nível compreende os conceitos mais complexos da ontologia. O conceito de policy define um arranjo de atores, atividades e objetos de valor organizados por relações de Teoria de Agência Múltipla. Cinco padrões de policies foram identificados por de Alencar Silva et al. (2017): single, double-check, chokepoint, committee e gossip. Esses padrões facilitam a modelagem de uma rede de valor semântica, e podem ser usados como estratégias de monitoramento preventivo de atividades de alto risco dentro da rede. Os atores, atividades e objetos de valor são conectados por atos de fala, e organizados segundo o Role-Based Access Control [Ferraiolo et al. 2001]. Um ponto de partida para estabelecer uma estratégia de monitoramento de atividade de risco é o arranjo no qual um regulador é relacionado a uma atividade via comprometimento de autoridade, sendo esta atividade conectada a um objeto de valor por meio de diferentes atos de produção. A ontologia descreve cada padrão por meio de uma concatenação de propriedades. O padrão single, descrito na Listagem 6 abaixo, ocorre sempre que o principal não delega nenhuma responsabilidade de monitoramento, e, portanto, necessita da autorização de regulador da rede para monitorar as atividades de agentes ou terceiros.

\section{Listagem 6. Lógica de descrição do padrão de agência single}

Class: Single

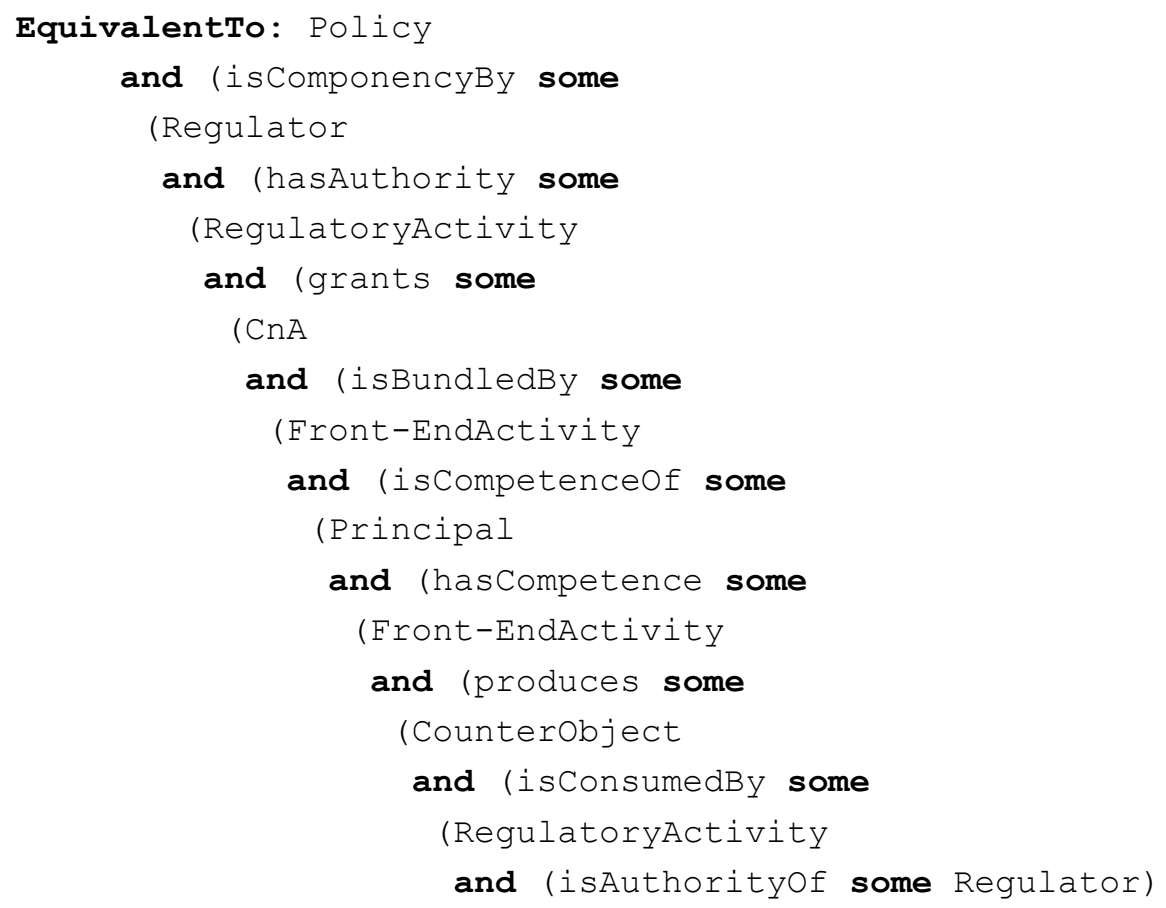


O padrão double-check (checagem dupla) ocorre sempre que o principal delega parcialmente a responsabilidade de monitoramento, ou seja, o principal assume a responsabilidade de monitoramento de terceiros juntamente com um agente da rede. Segundo a descrição lógica ilustrada na Listagem 7, o principal agrega um objeto de acreditação e certificação concedida pelo regulador e um objeto de prova de entrega de produto ou serviço concedida pelo terceiro. Além disso, um agente também agrega um objeto de certificação e acreditação e um objeto de prova do terceiro, que será analisado e distribuído em seguida para o principal. Dessa forma, o principal avalia as provas distribuídas pelo agente e as provas coletadas diretamente do terceiro.

\section{Listagem 7. Lógica de descrição do padrão de agência double-check}

Class: Double-Check

EquivalentTo: Policy

and (snv:iscomponencyBy some

(Regulator

and (hasAuthority some

(RegulatoryActivity

and ( grants some

( CnAObjects

and (isBundledBy some

(Front-EndActivity

and (iscompetenceof some

(Principal

and (hascompetence some

(Front-EndActivity

and (produces some

(CounterObject

and (isconsumedBy some

(RegulatoryActivity

and

(isAuthorityof

some

and (grants some

Regulator)) )) )) )) ) ) ) )

(CnAObjects

and (isBundledBy some

(ResourceActivity

and (iscompetenceof exactly 1 (Agent

and (hascompetence some

(ResourceActivity

and (distribute some

(CounterObject

and (isconsumedBy some

(RegulatoryActivity

and

(isAuthorityof

some

(Regulator) ) ) ) ) ) ) ) ) ) ) ) ) ) ) )

O padrão chokepoint (ou ponto de estrangulamento) ocorre sempre que o principal delega completamente a responsabilidade de monitoramento, ou seja, apenas os agentes 
assumem a responsabilidade de monitorar os terceiros. Segundo a descrição axiomática ilustrada na Listagem 8, no padrão chokepoint os agentes agregam objetos de acreditação e certificação do regulador e objetos de prova do terceiro. Em seguida, outro agente agrega todos os objetos de prova e concede ao principal. Esse arranjo de atores configura um "ponto de estrangulamento", através do qual um principal delega a responsabilidade de monitoramento para um único agente que agrega provas de múltiplos agentes.

\section{Listagem 8. Lógica de descrição do padrão de agência chokepoint}

Class: Chokepoint

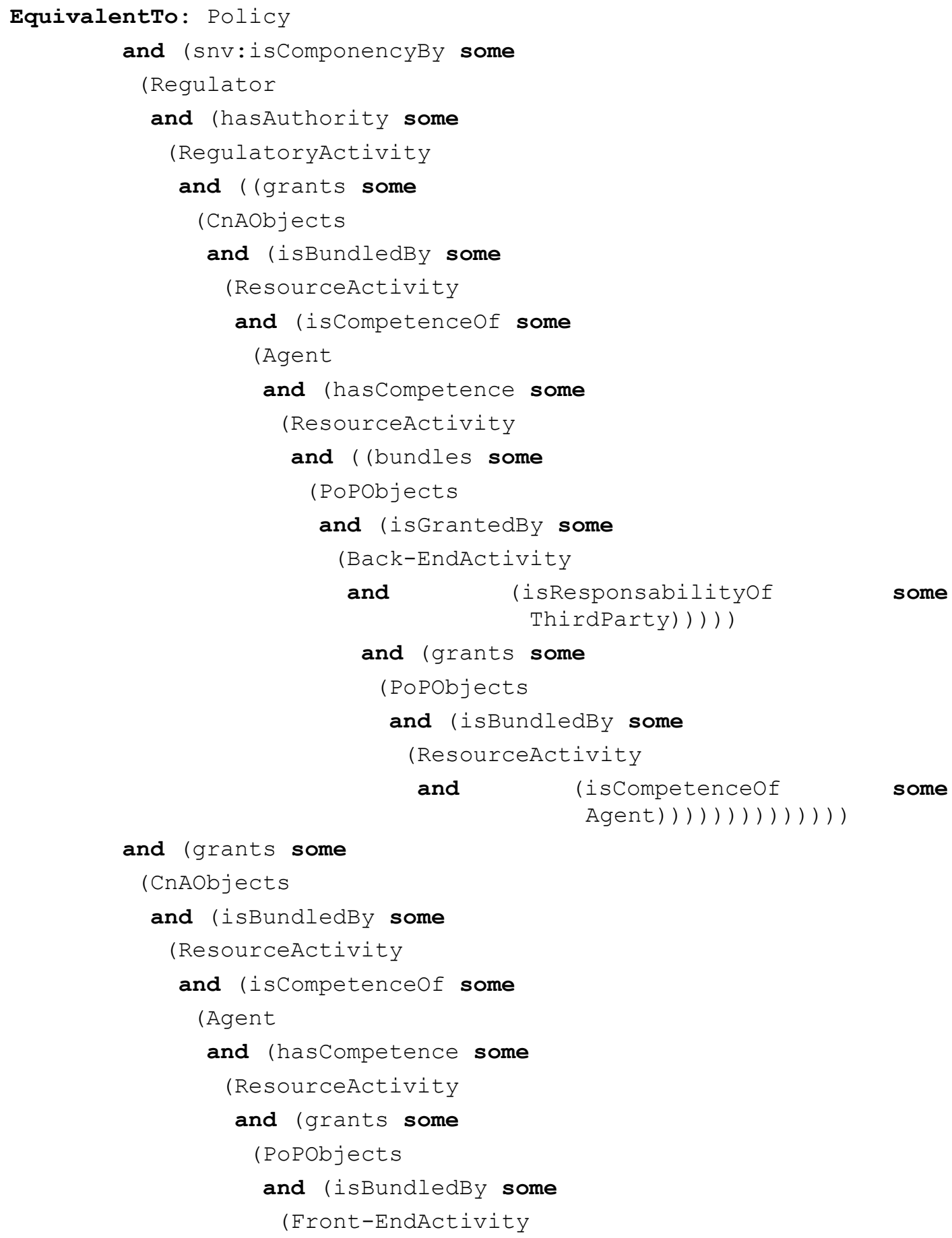


and

O padrão committee (comitê) ocorre sempre que o principal monitora uma atividade de risco e ainda delega parte da responsabilidade do monitoramento para outros dois agentes, formando um comitê de monitoramento realizado por três atores da rede. Segundo a descrição axiomática da Listagem 9, tanto o principal quanto os agentes agregam um objeto de acreditação e certificação concedido pelo regulador e um objeto de prova concedida pelo terceiro. Além do mais, os agentes concedem as provas ao principal. Dessa forma, principal realiza um comitê de monitoramento juntamente com os agentes.

\section{Listagem 9. Lógica de descrição do padrão de agência committee}

\section{Class: Committee}

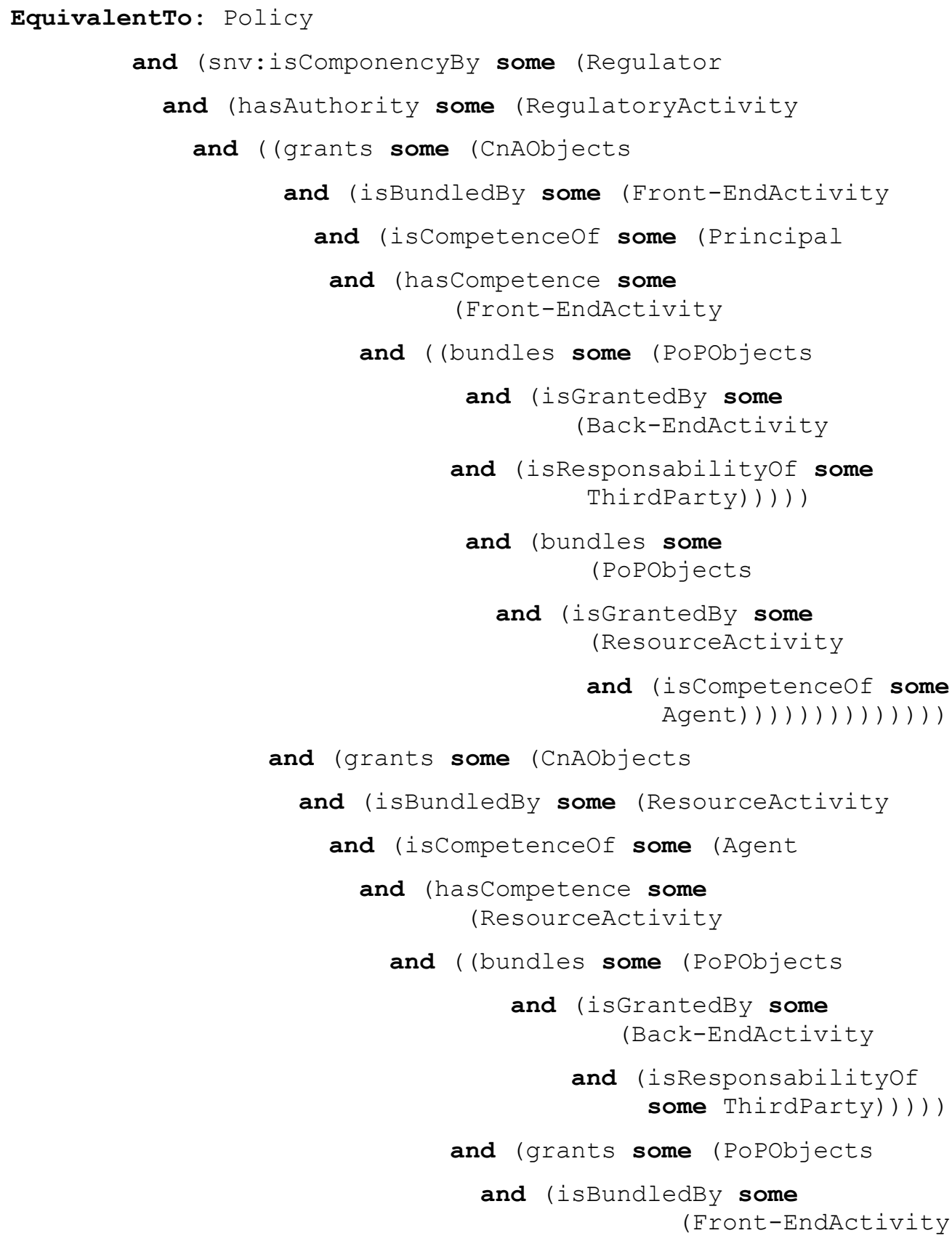




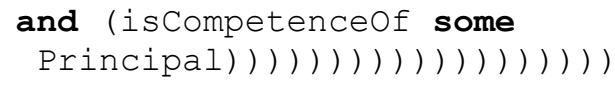

O padrão gossip (fofoca) ocorre sempre que o principal delega completamente sua responsabilidade de monitoramento, obtendo os objetos de provas válidos de vários caminhos da rede. Segundo a descrição axiomática da Listagem 10, no padrão gossip o principal recebe o objeto de prova de vários agentes que agregam objetos de prova do terceiro e concedem para outros agentes e para o principal. Dessa forma, o principal monitora o terceiro segundo as informações fornecidas pelos agentes.

Listagem 10. Lógica de descrição do padrão de agência gossip

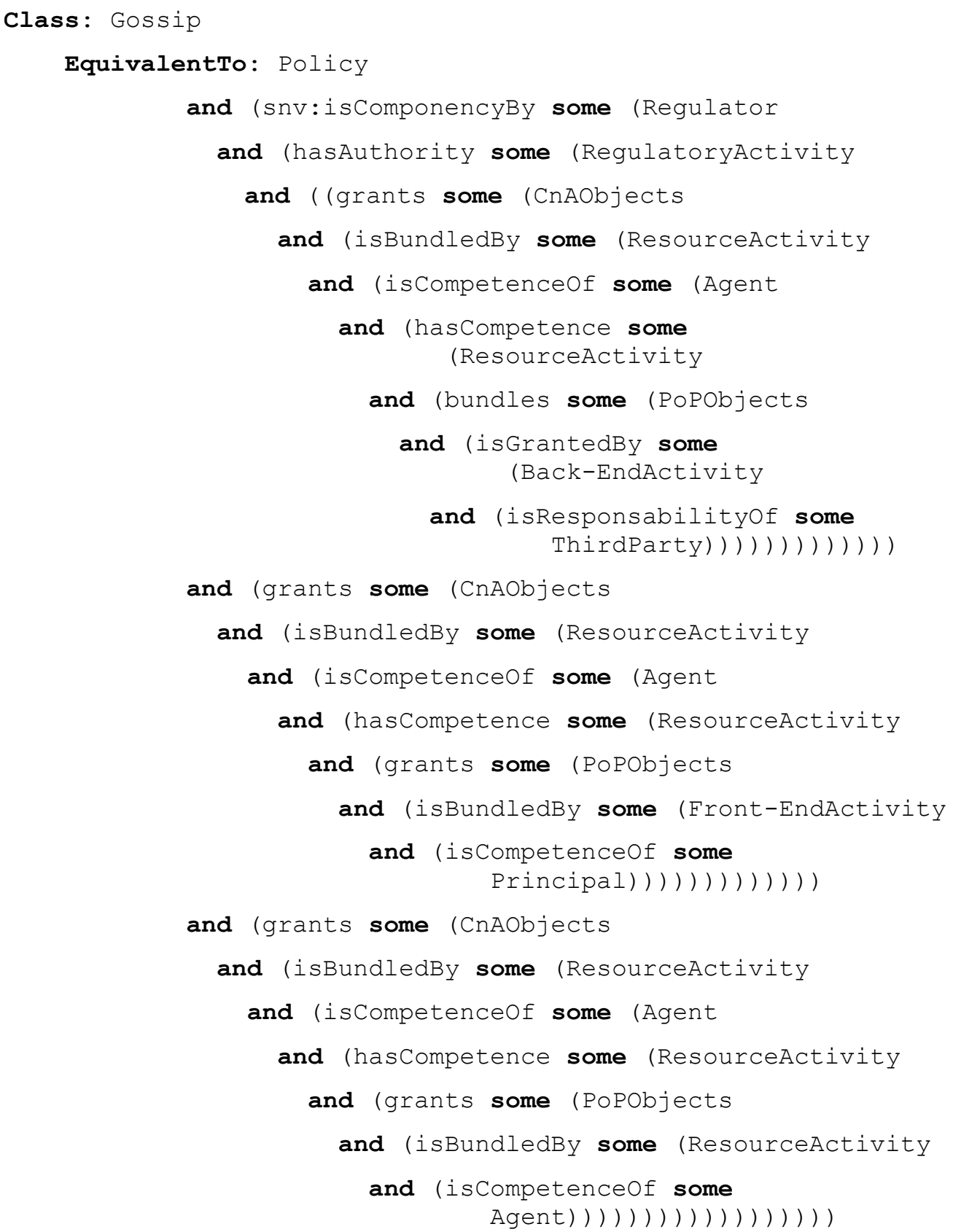

As transações de valor definem a troca de objetos de valor entre dois atores da rede. Uma transação assegura a sustentabilidade econômica da rede quando cada atividade realiza pelo menos uma transação de valor. $\mathrm{O}$ axioma descrito da Listagem 11 
três tipos de transações: a core object transaction, proof-of-performance object transaction e a certification and accreditation object transaction. Cada tipo de transação descreve o tipo de objeto que está sendo trocado entre os atores, e para cada transação há pelo menos um counter-object de retorno. As transações implementam o conceito de benefício e sacrifício, e por meio de regras realizam a inferência do tipo de objeto que está sendo trocado entre os atores. A Tabela 4 sumariza as regras SWRL que auxiliam na inferência de cada tipo de transação.

Tabela 4. Regras SWRL para classificação do tipo da transação

\begin{tabular}{|c|c|}
\hline $\begin{array}{c}\text { Tipos de } \\
\text { Transações } \\
\end{array}$ & Regras SWRL para atribuição das transações \\
\hline $\begin{array}{c}\text { Core } \\
\text { transaction }\end{array}$ & 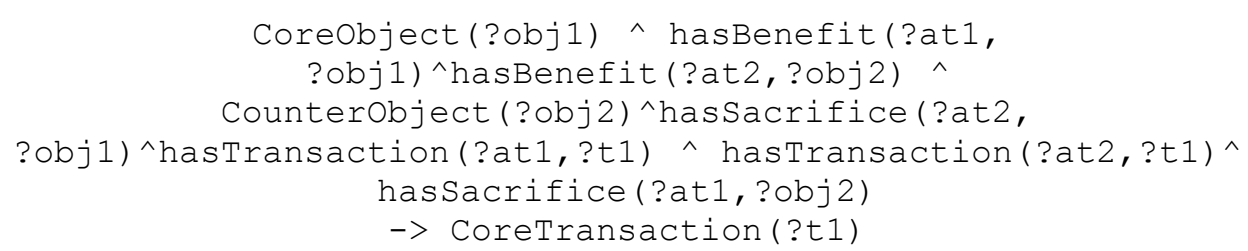 \\
\hline $\begin{array}{c}\text { PoP } \\
\text { transaction }\end{array}$ & 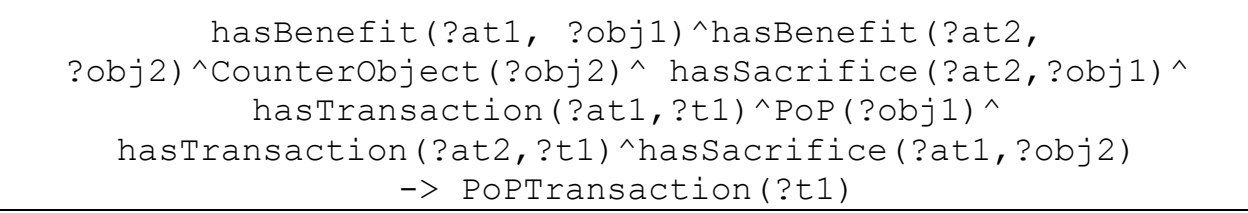 \\
\hline$\underset{\text { transaction }}{C n A}$ & 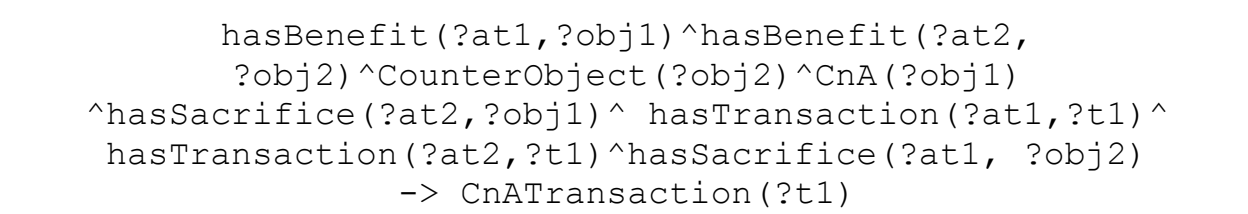 \\
\hline
\end{tabular}

Listagem 11. Lógica de descrição da classe ValueTransaction

Class: ValueTransaction

EquivalentTo: CnATransaction or CoreTransaction or PoPTransaction

No topo conceitual, uma necessidade de negócio define o ponto inicial de configuração de uma rede de valor. Qualquer modelo de negócio nasce da necessidade de um determinado cliente. Tal necessidade é uma demanda do ator com o papel de principal na rede segundo a segundo a Service-Dominant Logic, definida por Vargo e Akaka (2009). A necessidade de negócio é satisfeita pela comunicação das proposições de valor por meio de transações de cooperação entre os atores da rede. Além disso, a satisfação de uma necessidade de negócio depende do valor projetado para o produto ou serviço, e portanto, valida o arranjo de uma rede de valor. O valor projetado é composto de partições disjuntas de value surplus, value balance e value shortage [Steedman 1975]. Em esvalue, a noção de necessidade de negócio é reificada como um objeto de valor desejado pelo consumidor. Aqui, essa noção é estendida pela separação da identidade de um objeto principal de negócio (core-object) de seu indicador de valor, que pode ser objetivo (p. ex., quantidade, qualidade, tempo e localização) ou subjetivo (p. ex., privacidade, confiabilidade ou confiança). Um objeto principal de negócio atende às necessidades de um consumidor quando seu sacrifício é menor do que o seu benefício, que é medido pela quantificação dos recursos monetários pagos em troca do produto ou serviço fornecido 
pela rede. A definição axiomática de uma necessidade de negócio é apresentada na Listagem 12.

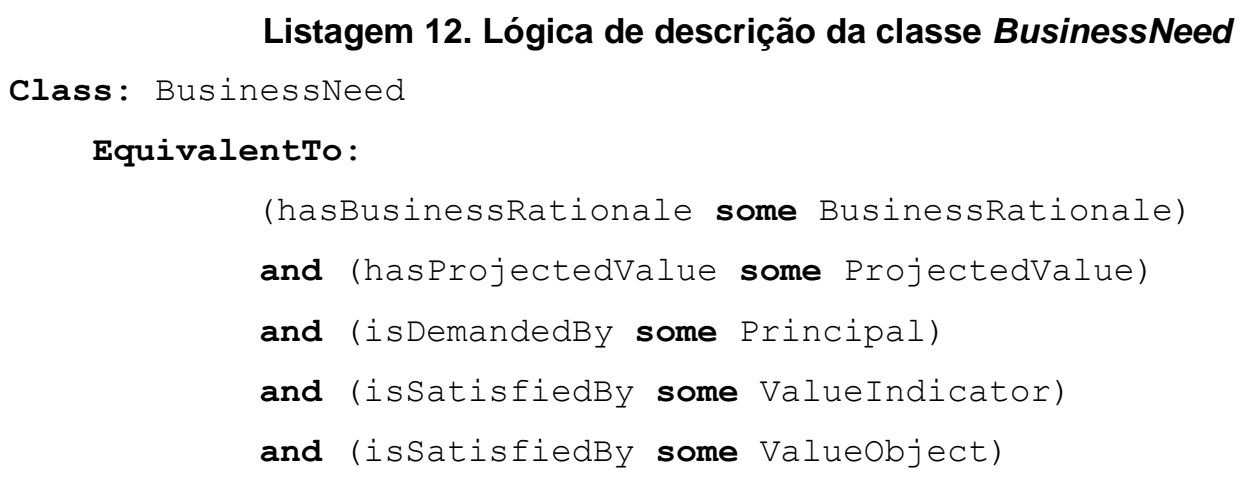

A ontologia proposta tem o objetivo de mitigar o problema da configuração semiautomática de um modelo de rede de valor. Isso justifica o uso da linguagem OWL para representação de máquina, traduzida neste artigo para a sintaxe Manchester de OWL, de modo a facilitar a compreensão humana. Quanto ao processo de Engenharia de Ontologia, foi usada a metodologia proposta por Sure et al. (2009) pela formalidade de seu processo, o qual inclui ciclos iterativos de análise, especificação, validação e avaliação da ontologia.

\subsection{Avaliando a ontologia}

A avaliação é a tarefa de mensurar a qualidade de uma ontologia [Vrandecic 2009]. Para avaliar a ontologia proposta, foram utilizados critérios de verificação, validação e avaliação proposta por Gómez-Pérez (2004). No processo de verificação pode-se avaliar a corretude, completude e consistência da ontologia. Neste trabalho foram utilizados os motores de inferência Pellet, Fact++ e Hermit para verificação de consistência da ontologia. A corretude e completude foram verificadas por meio de questões de competência respondidas pela ontologia utilizando consultas formuladas na linguagem SPARQL.

A segunda etapa do processo de verificação propõe uma verificação guiada pelas questões de competências [Grüninger and Fox 1995]. Nesta etapa, as questões de competência apresentadas no documento de especificação de requisitos da ontologia devem ser formalizadas em uma linguagem de consulta que possa ser usada pela ferramenta que vai usar a ontologia [Vrandecic 2009]. As seis questões de competência são respondidas usando a linguagem de consulta SPARQL e são apresentadas nas tabelas a seguir.

A primeira consulta corresponde às responsabilidades dos atores da rede, ou seja, esta consulta visa identificar quem são os atores que compõem uma determinada rede de valor e o seu papel na rede. A Tabela 5 apresenta a consulta SPARQL para resolução desta questão de competência. Como resultado, podem ser obtidos o ator, o identificador e o seu papel na rede. 


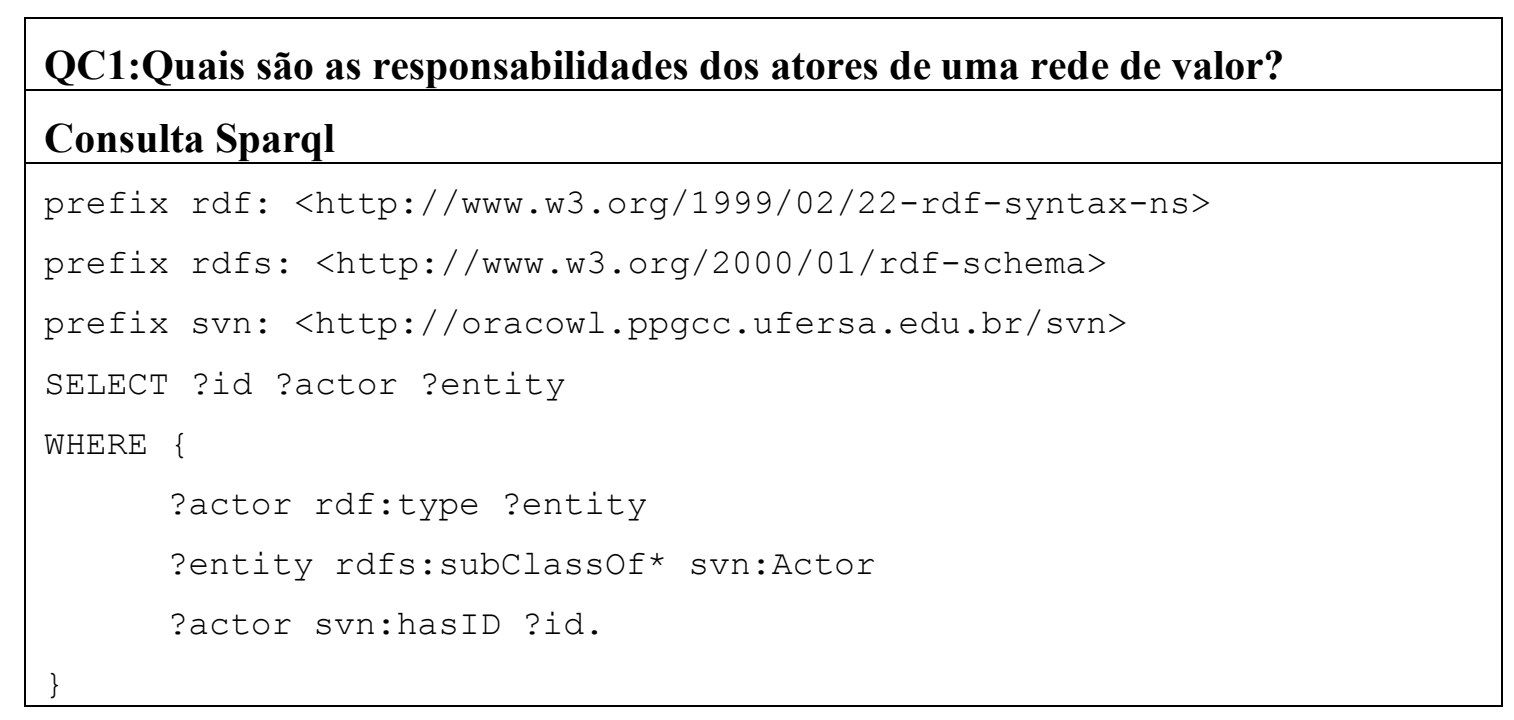

A segunda consulta corresponde às atividades realizadas pelos atores da rede, ou seja, esta consulta visa identificar quais atividades cada ator tem a competência, responsabilidade ou autoridade de realizar. A Tabela 6 apresenta a consulta SPARQL para resolução desta questão de competência. Como resultado, pode ser obtido o ator, a atividade e o tipo da atividade.

Tabela 6. Consulta SPARQL para resolução da segunda questão de competência da ontologia

\section{QC2: Quais são os tipos de atividades realizadas pelos atores da rede?}

\section{Consulta Sparql}

prefix rdf: <http://www.w3.org/1999/02/22-rdf-syntax-ns>

prefix rdfs: <http://www.w3.org/2000/01/rdf-schema>

prefix svn: <http://oracowl.ppgcc.ufersa.edu.br/svn>

SELECT ?id ?actor ?eof ?activity ?entity

WHERE \{

?actor rdf:type ?type.

?activity rdf:type ?entity.

?type rdfs:subclassof* svn:Actor.

?entity rdfs:subclassof* svn:ValueActivity.

?actor ?eof ?activity.

?actor svn:hasid ?id.

filter(!strstarts (str(?eOf), str(svn:active_EOF))).

filter(!strstarts (str(?entity), str(svn:ValueActivity))) .

\}

A terceira consulta corresponde aos objetos de valor comunicados entre os atores, ou seja, esta consulta visa identificar quais objetos estão sendo trocados entre os pares de atores da rede. A Tabela 7 apresenta a consulta SPARQL para resolução desta questão de competência. Como resultado, pode ser obtido os atores, as atividades e os objetos de valor. 
Tabela 7. Consulta SPARQL para resolução da terceira questão de competência da ontologia

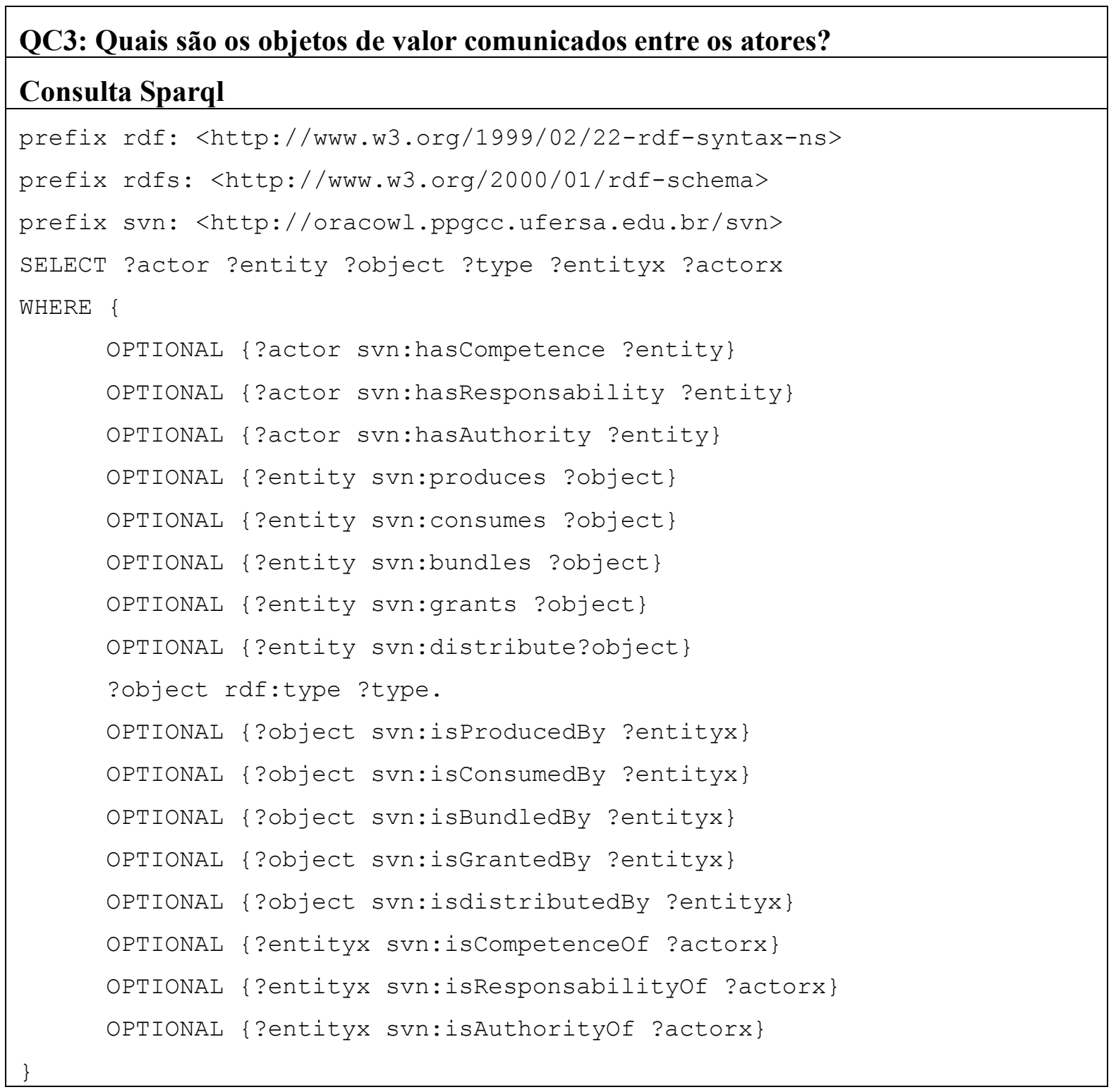

A quarta consulta corresponde às políticas organizacionais da rede de valor. Estas políticas são derivadas de padrões de monitoramento de agência. Desse modo, pode-se buscar o objeto a ser monitorado e o padrão de agência que está sendo utilizado. A Tabela 8 apresenta a consulta SPARQL para resolução desta questão de competência.

Tabela 8. Consulta SPARQL para resolução da quarta questão de competência da ontologia

QC4: Como recuperar políticas organizacionais que compõem uma rede de valor? 


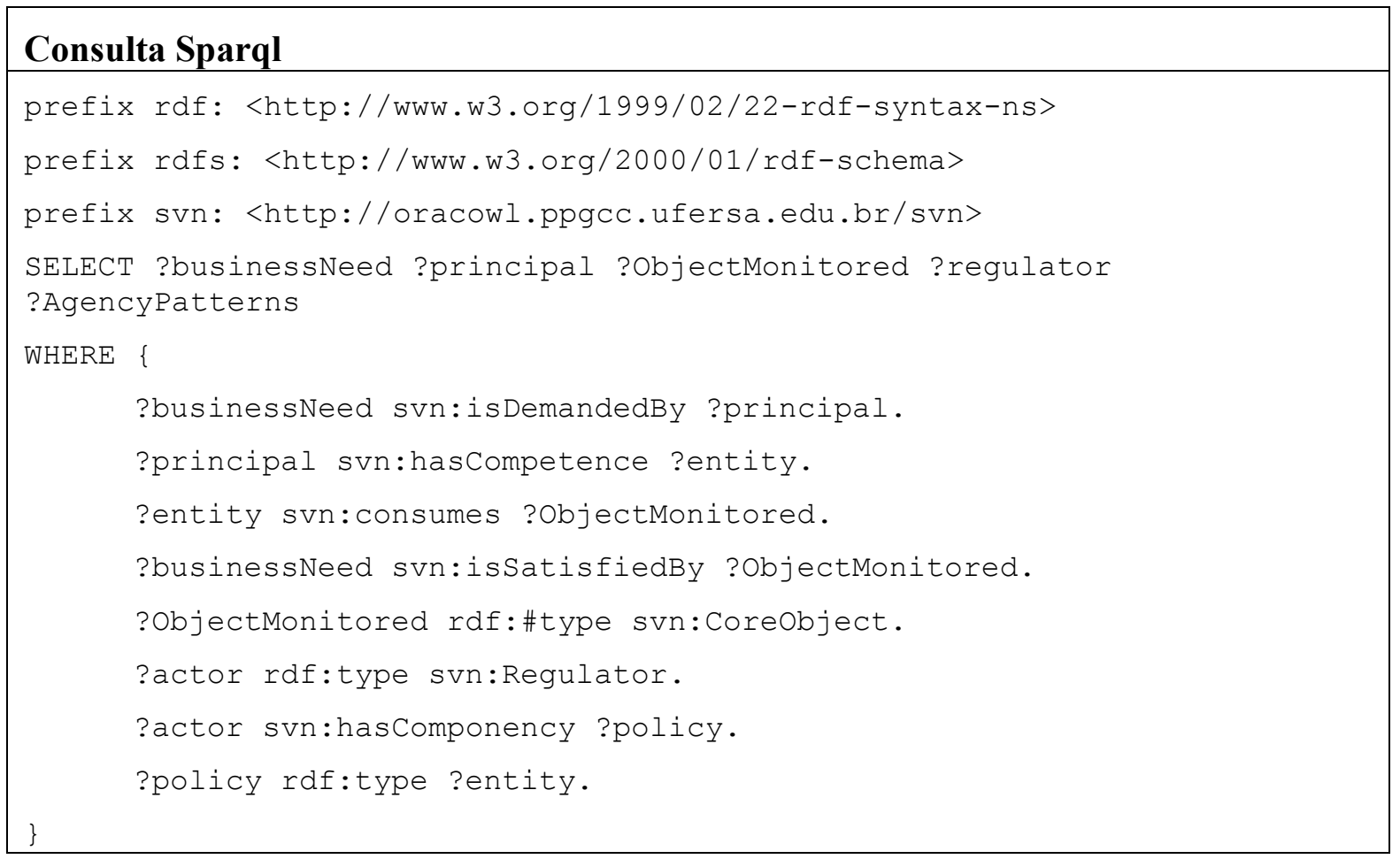

A quinta consulta permite verificar se as transações da rede são economicamente sustentáveis. Para isso, é necessário verificar se para cada sacrifício é identificado um benefício correspondente. A Tabela 9 apresenta a consulta SPARQL para resolução desta questão de competência.

Tabela 9. Consulta SPARQL para resolução da quinta questão de competência da ontologia

\section{QC5: Como verificar se transações da rede são economicamente sustentáveis (reciprocidade econômica)?}

\section{Consulta Sparql}

prefix rdf: <http://www.w3.org/1999/02/22-rdf-syntax-ns>

prefix rdfs: <http://www.w3.org/2000/01/rdf-schema>

prefix svn: <http://oracowl.ppgcc.ufersa.edu.br/svn>

SELECT ?id ?actor ?entity ?object ?objectx ?entityx ?actorx

WHERE \{

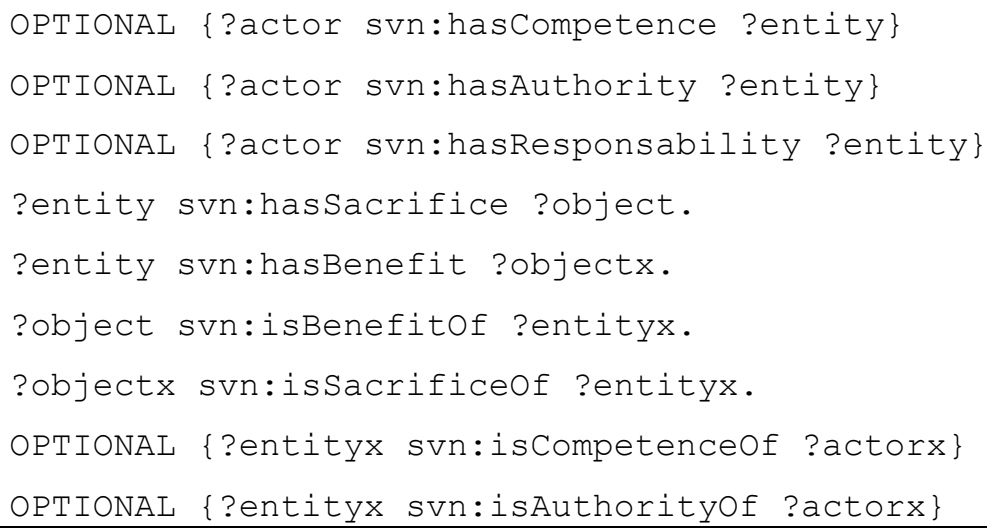


OPTIONAL \{?entityx svn:isResponsabilityOf ?actorx\}

\}

Na sexta consulta pode-se identificar o valor mensurável da rede. Este valor é definido por meio da análise de valores objetivos e subjetivos, permitindo assim identificar se a rede satisfaz a necessidade de negócio do principal. A Tabela 10 apresenta a consulta SPARQL para resolução desta questão de competência.

Tabela 10. Consulta SPARQL para resolução da sexta questão de competência da ontologia

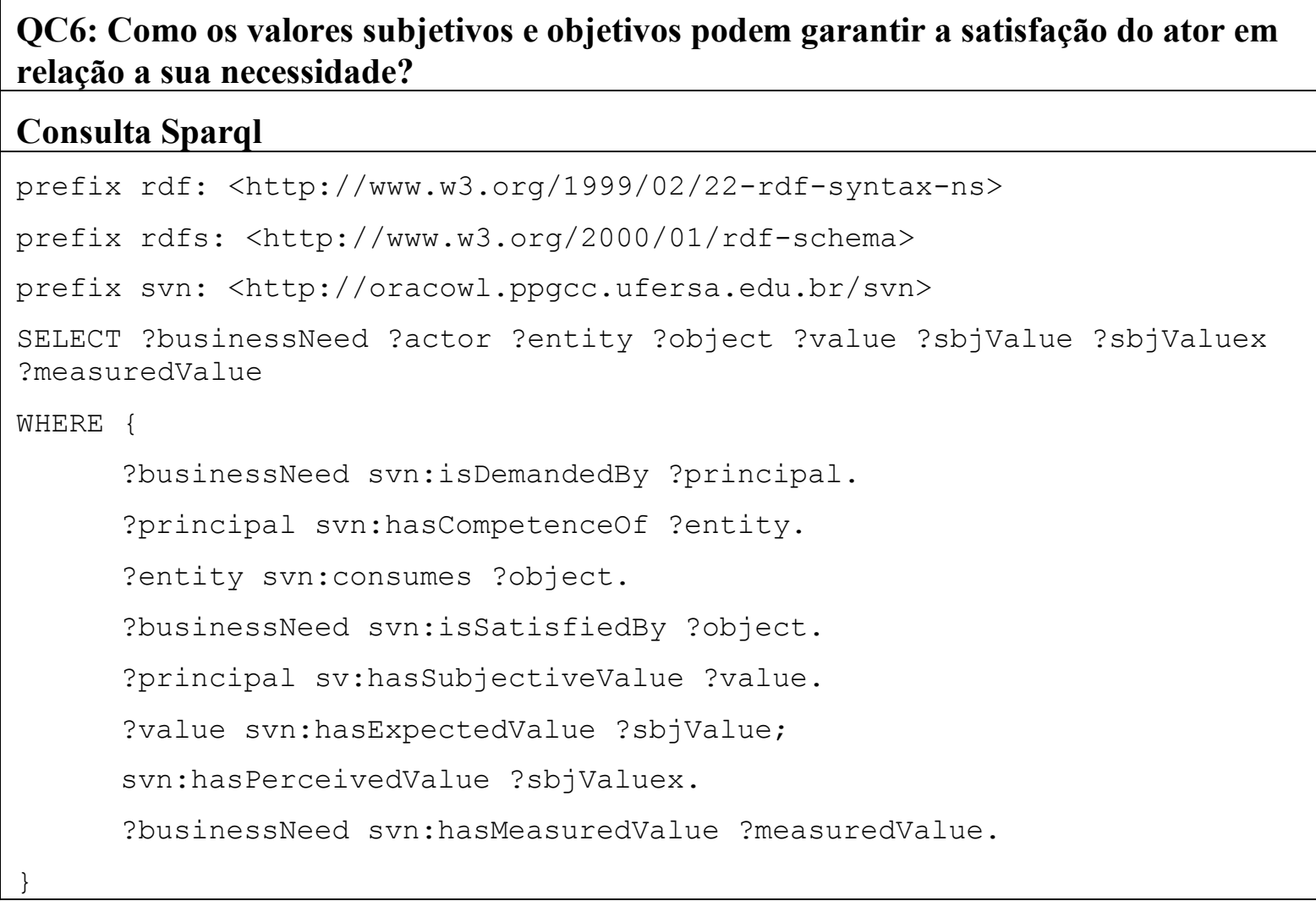

A Seção 4 a seguir apresenta o processo de validação, na qual foram utilizados cenários de estudos de caso reais reportados na literatura de modelagem de redes de valor com ezvalue. As consultas acimas foram usadas com objetivo de extrair partes da rede de valor e assim apresentá-las ao usuário como um grafo.

\section{Estudo de Caso Observacional}

De acordo com a metodologia de Engenharia de Ontologias adotada nesta pesquisa, esta etapa segue o processo de aplicação e evolução da ontologia, e segundo a Design Science pode incluir um estudo de caso. A pesquisa de estudo de caso é um método abrangente e a escolha apropriada do projeto de estudo é essencial para o sucesso da validação. Estudos de caso se dividem em duas grandes categorias: observacionais e intervencionais. De acordo com Wieringa (2014b), um estudo de caso observacional é a análise de um caso real sem intervenção direta do pesquisador no caso. Assim, o estudo de caso observacional é um caso já reportado na literatura que pode ser usado para avaliar a implementação de um sistema de informação. 
Para aquisição dos objetos de estudo foram realizadas pesquisas em busca dos indivíduos (cenários de uso) que satisfizessem o predicado da população na literatura de esvalue. Três cenários de uso foram selecionados. O primeiro caso apresenta um cenário de caso em Direitos de Propriedade Intelectual [Gordijn et al. 2011]; o segundo cenário provém de caso em medição inteligente de energia renovável [de Alencar Silva et al. 2017]; o terceiro compreende um cenário de caso em controle aduaneiro [de Alencar Silva 2013]. Estes cenários possuem o objetivo de responderem às seguintes questões: todos os elementos do modelo de rede de valor são instanciados corretamente na ontologia? A ontologia infere conhecimento a partir da rede instanciada?

Para validade destes casos, foi utilizada a inferência analógica. A inferência analógica consiste em descobrir generalizações, buscando a similaridade entre os objetos de estudo [Wieringa 2014b]. Portanto, o objetivo da exploração destes casos foi identificar generalizações nestes objetos de estudo de forma que possam refinar e validar a ontologia. A estratégia de indução analítica utilizada no processo de amostragem dos casos inclui validação dos níveis conceituais da ontologia (vide Seção 3). Cada cenário foi utilizado para validar uma parte da ontologia. A adequabilidade e as limitações dos cenários foram identificadas com base nas respostas às questões de competência obtidas pelo uso da ontologia proposta.

\subsection{Cenário de Direito de Propriedade Intelectual}

O cenário de direito de propriedade intelectual apresentado por Gordijn et al. (2011) trata da regulação do direito de reprodução de música em locais públicos em alguns países da Europa. O cenário de uso abordado neste caso, trata especificadamente do modelo de negócios da Holanda. $\mathrm{O}$ caso aborda que, se um supermercado, uma cafeteria ou outros locais públicos desejarem tocar faixas de músicas em seus estabelecimentos, devem pagar aos proprietários os direitos sobre a reprodução daquele material.

O cenário inicia com um consumidor que deseja reproduzir uma faixa de música em seu estabelecimento comercial. Para satisfazer sua necessidade de negócio, atores intermediários como as sociedades de Direito de Propriedade Intelectual - DPI atuam juntamente com provedores de música digital para atingir esse objetivo [Gordijn et al. 2011]. As sociedades DPI são responsáveis por coletar o dinheiro dos consumidores e distribuir aos respectivos segmentos artísticos depositários da propriedade intelectual, os quais incluem artistas, produtores, editores, compositores e instrumentistas.

Como parte da estratégia de indução analítica, neste caso será apresentado a instanciação do primeiro nível da ontologia. As relações entre os elementos da ontologia podem ser vistas no grafo ilustrado na Figura 2. 


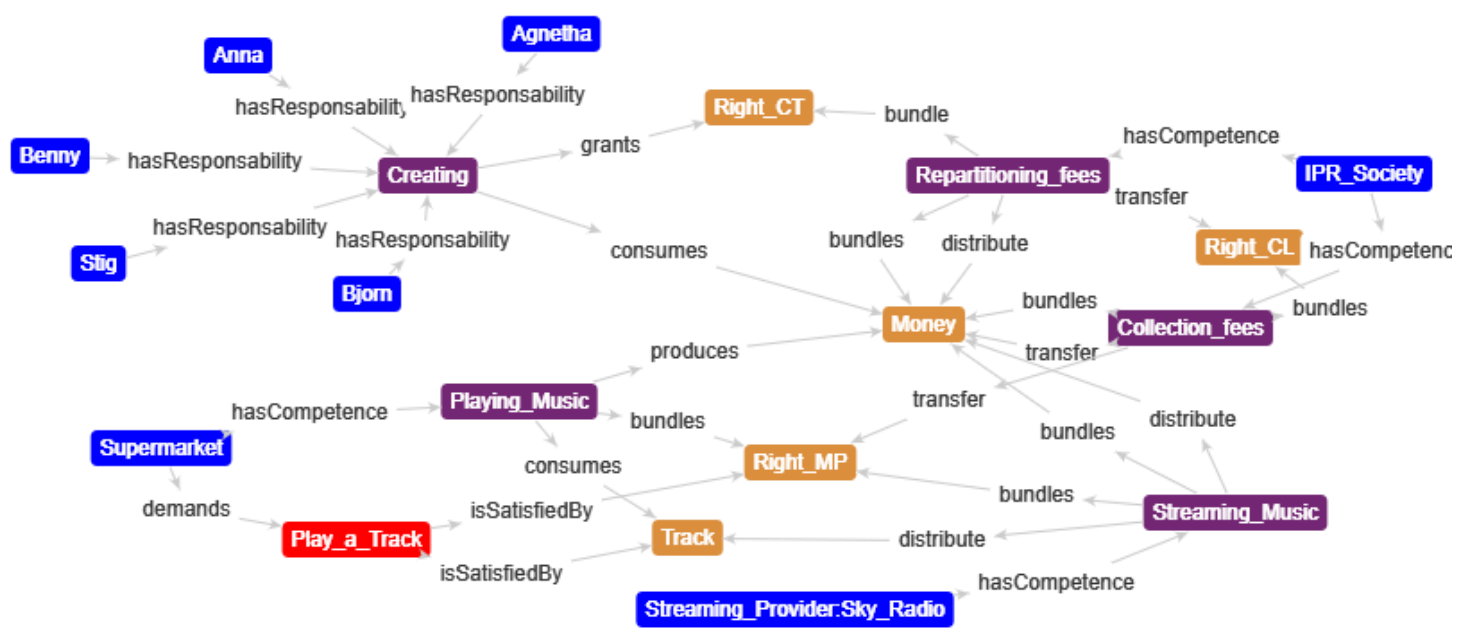

Figura 2. Visualização da rede de valor semântica do cenário de Direito de Propriedade Intelectual (com foco nos atores, atividades de negócio e objetos de valor)

A necessidade de negócio da rede (conceito destacado em vermelho) é tocar faixas de músicas. Esta pode ser uma necessidade de negócio de um supermercado, por exemplo, operando como o principal (conceito destacado em azul) da rede. Sua necessidade é satisfeita por dois objetos de valor (conceito destacado em laranja): as faixas de músicas (tracks) e os direitos de tornar as faixas públicas (Right_MP), classificadas como objeto principais do negócio. Dessa forma, o supermercado obteria a competência para realizar a atividade de tocar músicas em seu estabelecimento. Os demais atores estão conectados às suas respectivas atividades de valor via competência, autoridade ou responsabilidade, enquanto que as atividades alteram o estado do objeto com atos de produção. Dessa forma, é possível visualizar uma nova perspectiva da interação entre os elementos da rede trazendo mais sentido aos componentes do modelo. Todos os elementos do modelo foram instanciados e classificados corretamente pela ontologia, finalizando a primeira etapa da indução analítica.

Este estudo de caso auxiliou no refinamento das relações da tripla ator-atividadeobjeto. Foram ainda refinadas as relações principal-agente, agente-agente e agenteterceiro, mediante as quais esses atores produzem, consomem, agregam ou distribuem objetos de valor, e como estas propriedades alteram o estado ou indicadores de valor atribuídos a esses objetos.

\subsection{Cenário de Medição Inteligente de Energia}

O segundo cenário de estudo de caso selecionado foi apresentado em de Alencar Silva e Weigand (2011), de Alencar Silva et al. (2017) e posteriormente em da Silva Reis et al. (2018). O cenário é uma projeção futura do mercado de energia liberalizado na Europa, normalizado pela Diretiva 2009/72/CE da União Europeia [EU 2009]. Nesse cenário, os proprietários terão a opção de escolher não só os fornecedores de energia, mas também as empresas de medição inteligente que atendam melhor às suas necessidades.

Nesse modelo de rede de valor, o consumidor final é uma pessoa física ou jurídica com residência fixa, representada por um segmento de mercado de Agentes de Balanceamento (Balance Responsible Parties - BRPs). Os relatórios da UE revelaram 
que os principais entraves à adoção de soluções de medição inteligente pela população europeia dizem respeito à privacidade, ou seja, a informação sobre o consumo de energia pode ser explorada oportunisticamente [European 2014]. Assim, o consumidor final pode considerar a avaliação de outros atores a fim de verificar a privacidade fornecida por um serviço tão inovador, antes de firmar acordo de serviço com um operador de medição.

Como parte da estratégia de indução analítica, este cenário apresenta características para validar os aspectos qualitativos da ontologia, mais especificamente os conceitos de valor objetivo e subjetivo de um produto ou serviço. $\mathrm{O}$ valor subjetivo em análise neste cenário é a privacidade sobre a informação individual do consumo de energia. Os medidores inteligentes possuem valores objetivos a serem analisados também pelo BRP que não serão ilustrados na descrição deste cenário, pois o foco aqui é como os atores comunicam diferentes percepções de qualidade do serviço. Neste caso, a necessidade de negócio do consumidor pode ser satisfeita por um serviço agregado de energia e relatório de medição, cuja produção depende do medidor inteligente, acreditação e certificação de medição, auditoria do dispositivo e avaliação da privacidade reportada por vários atores, conforme ilustrado na Figura 3.

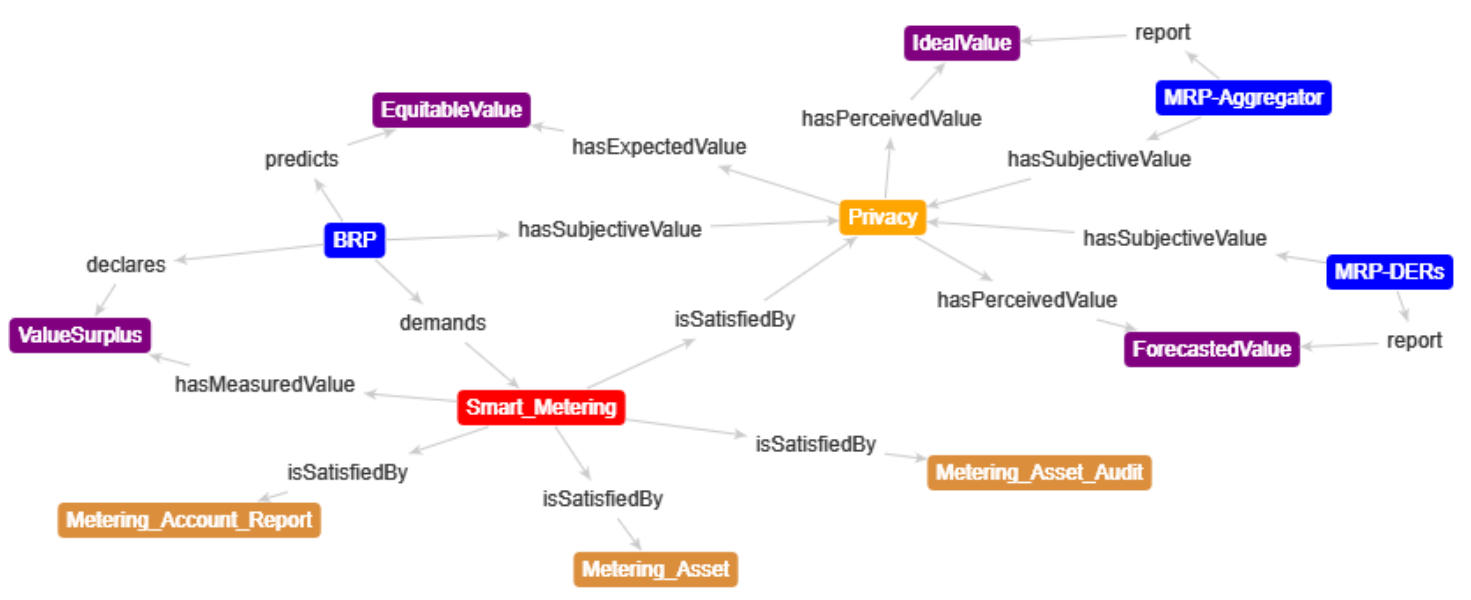

Figura 3. Visualização da rede de valor semântica do cenário de medição inteligente de energia, com foco na necessidade de negócio e valores subjetivos

Para o BRP declarar uma falta, equilíbrio ou excedente de valor, primeiro devese prever uma aferição esperada para este valor subjetivo. Em seguida, pode-se confrontar este valor com as aferições de valor experimentado reportadas por agentes ou por reguladores da rede. Um conjunto de regras SWRL definidas para a ontologia comparam valor esperado e valor experimentado atribuídos, utilizando a escala SERVQUAL, além da declaração de valor projetado para satisfação da necessidade de negócio. Neste cenário, a privacidade como valor subjetivo apresentaria um excedente de valor como valor projetado para satisfação da necessidade de negócio.

O valor esperado (IdealValue) e o valor percebido (ExperiencedValue) ajudam a definir uma análise qualitativa que serve de critério de avaliação da sustentabilidade da rede. Assim, a necessidade de negócio da rede pode ser analisada em cada ciclo da instanciação de atores da rede. 


\subsection{Cenário de Controle Aduaneiro}

Este cenário de uso corresponde a um caso do setor aduaneiro apresentado inicialmente em Bukhsh \& Weigand (2011) e posteriormente em de Alencar Silva (2013). Por ser um cenário de projeto organizacional, foi essencial para análise de permutas de objetos entre os atores da rede. Como parte da estratégia de indução analítica, este cenário proporciona uma validação do conceito de transações apresentado na ontologia. Para garantir a reciprocidade econômica na troca de valores entre dois ou mais atores em um modelo de negócio, é preciso garantir que para cada benefício tenha um sacrifício em troca [Lapierre 2000].

A necessidade de negócio deste cenário é constituída de mercadorias do comércio varejista de sucos naturais. A extremidade da rede é composta por grandes produtores de frutas. Para operar no varejo, o produtor precisa pagar primeiramente o imposto sobre bens de consumo em troca do documento de legitimação que o permite comercializar o seu produto com a indústria de processamento. Esta transação é apoiada por companhias de navegação que fornecem o serviço de transporte em troca de dinheiro. A indústria de processamento também precisa pagar os impostos sobre bens de consumo em troca do documento de legitimação para comercializar a matéria prima pré-processada. Esta atividade também é apoiada pelas companhias de navegação. Para a indústria de varejo satisfazer sua necessidade, deve obter o documento de legitimação da autoridade de controle aduaneiro e os bens acabados direto da indústria de transformação ou das companhias de navegação.

É válido ressaltar que as relações de benefício e sacrifício definem as transações entre os atores da rede. Como parte da indução analítica, as instanciações das transações são apresentadas em duas etapas. Na primeira, ilustrada na Figura 4, é possível identificar os benefícios e os sacrifícios realizados por cada ator da rede. De acordo com Lapierre (2000), as transações de negócios são constituídas de benefícios e sacrifícios, dependendo do ponto de vista de cada ator. Na transação entre a indústria de varejo e a indústria de transformação, o que é um sacrifício para a indústria de transformação (neste caso, bens acabados) é um benefício para indústria de varejo, por satisfazer a sua necessidade de negócio. Analisando este modelo de negócio do ponto de vista da troca de valor entre os atores da rede, pode-se identificar que para se concretizar uma transação de valor são necessários objetos de retorno. 


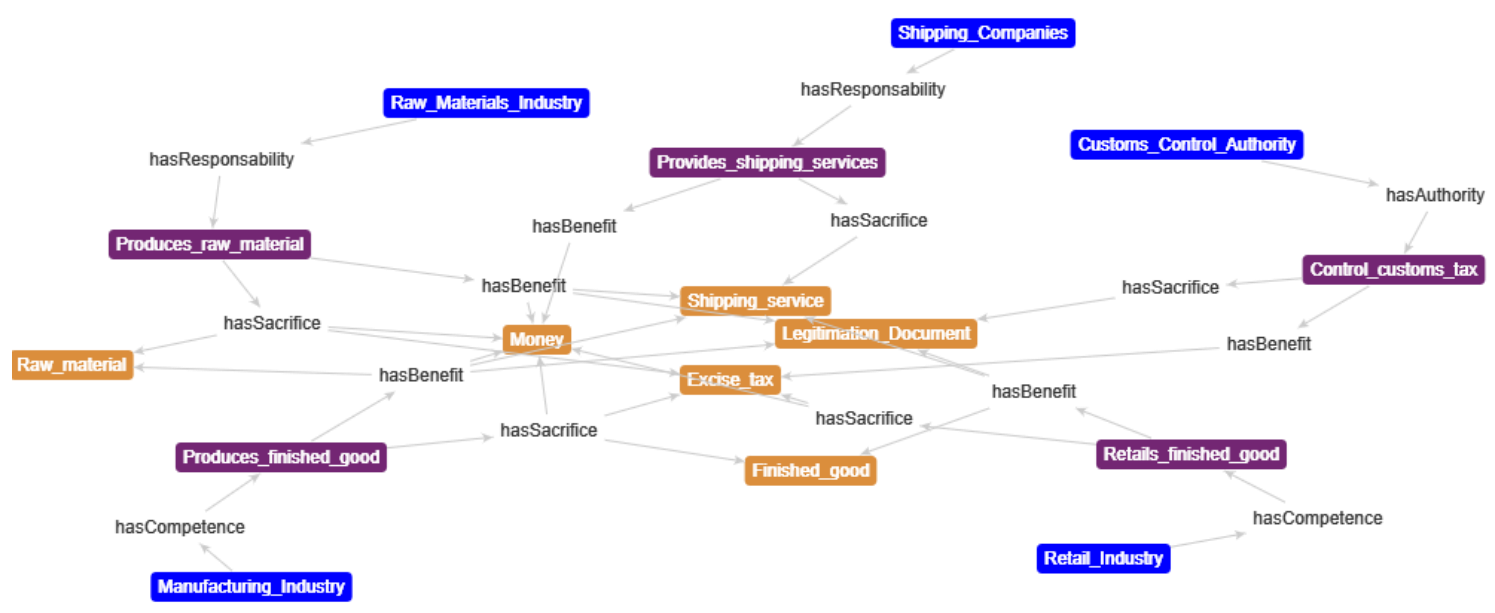

Figura 4. Visualização da rede de valor semântica do cenário de controle aduaneiro, com foco nas relações entre atores, atividades e objetos de valor

As transações são classificadas em três categorias distintas de acordo com o tipo de objeto de valor que comunicam. As transações podem ser de um dos tipos: Core Transaction, PoP Transaction ou CnA Transaction. A indústria de produção de frutas entrega a matéria-prima em benefício do dinheiro distribuído pela indústria de processamento. Por se tratar de uma transação envolvendo um objeto principal do negócio, a ontologia identifica que se trata de uma core transaction, conforme ilustrado na Figura 5. Essas transações só podem ser validadas na ontologia se houver reciprocidade econômica entre os atores, ou seja, se houver um objeto de retorno. Caso não o haja, a transação não será validada.

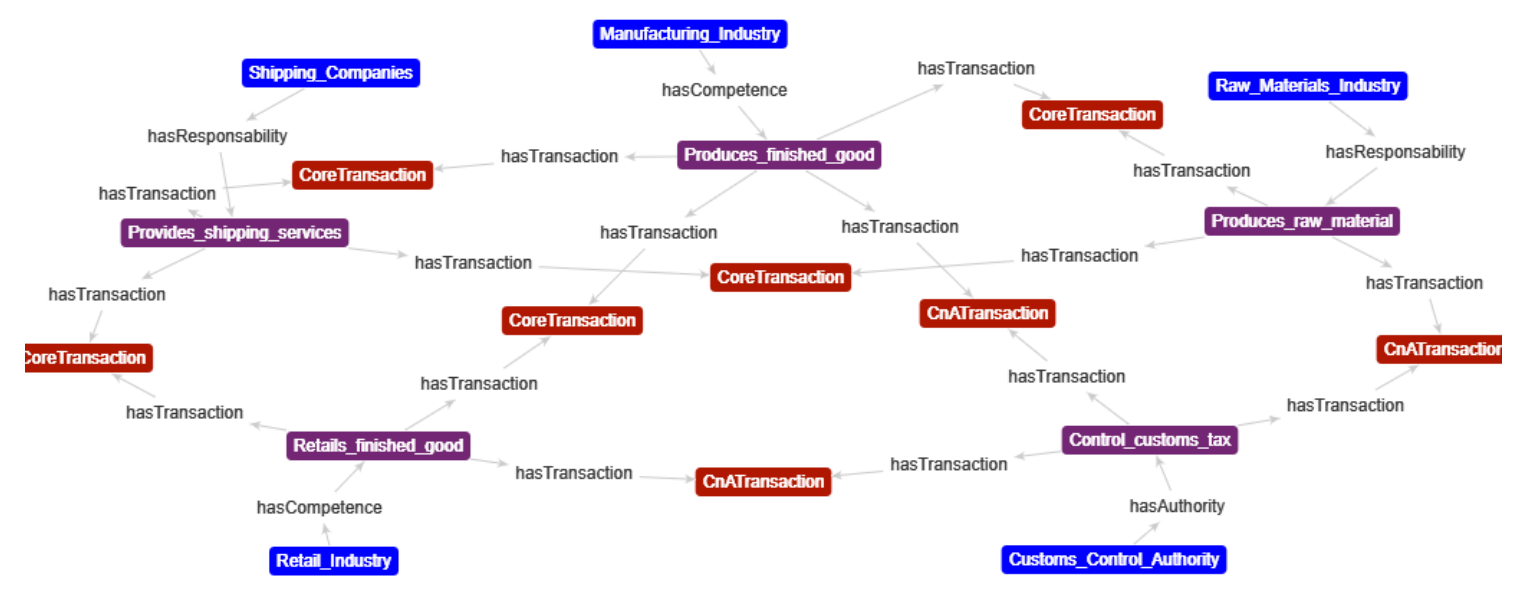

Figura 5. Visualização em grafo da rede de valor semântica do cenário de controle aduaneiro, com foco nas atividades e transações de valor

\subsection{Discussão}

A validação teórica da ontologia de redes de valor semânticas, foi realizada analisando o artefato por meio de cenários de uso. Para isso, foram utilizadas a 
metodologia de estudo de caso observacional, proposta pela design science, seguindo um protocolo de estudo de caso. De acordo com a metodologia de especificação da ontologia, está etapa corresponde a avaliação focada na ontologia que apresenta como resultado a ontologia alvo, além da sua aplicação e evolução.

Os estudos de caso observacionais auxiliaram no refinamento, permitindo assim elaborar axiomas que relacionassem corretamente cada conceito presente na SVNO. Esse elemento foi crucial para separar os conceitos de atores, atividades, objetos de valor, valores objetivos e subjetivos, e necessidade de negócio da rede. Com o estudo de caso observacional foi possível utilizar cenários já reportados na literatura para instanciar a ontologia. Todos os elementos presentes em cada cenário foram inseridos na ontologia e em seguida uma propriedade foi definida para o identificar na rede. Esse foi o ponto de partida para o motor de inferência classificar e organizar os elementos da rede de valor. Portanto, após a aplicação do motor de inferência novos conceitos foram inferidos, fornecendo novas informações que podem ser utilizadas por analistas de negócios. Essas informações podem ser sobre transações, políticas de organização da rede ou sobre valores objetivos e subjetivos dos objetos de valor, que podem ser previamente avaliados por outros atores. Além do mais, os analistas podem identificar, por exemplo, a importância de certas atividades ou objetos de valor pela quantidade de relações que elas possuem com outros elementos do modelo, identificando a sua importância na logística organizacional da rede, permitindo assim elaborar novas estratégias de negócio.

Quando um determinado ator é inserido na rede é possível definir o seu papel dentro da rede de valor e como ele poderá se relacionar com os demais atores presentes. Portando, relacionar este ator com as atividades necessárias para o pleno funcionamento da rede e quais objetos de valor são do seu interesse permite que eles elaborem contratos de parcerias entre si. Estes contratos são o ponto inicial para uma transação de valor. Em cada transação, é verificada a sua sustentabilidade de forma a garantir que a rede seja sustentável a todos os membros envolvidos. Portanto, os cenários apresentaram que todos os elementos do modelo podem ser instanciados e classificados corretamente na ontologia proposta. A inferência fornece conhecimento sobre estes elementos, e as consultas podem auxiliar na extração desse conhecimento.

O trabalho pode ser classificado em duas categorias: (1) um mecanismo de apoio à decisão - pois ainda não tem todos os componentes de um SAD (p.ex. uma interface gráfica intuitiva), mas apenas uma lógica de representação, produção e acesso ao conhecimento (i.e. ontologia, regras e padrões de consultas segundo o ORSD); e (2) um sistema orientado ou dirigido por ontologia, o qual tem uma ontologia de processo como seu fundamento, derivada de várias teorias usadas em Enterprise Engineering.

\section{Trabalhos Relacionados}

Esta seção apresenta uma discussão sobre os trabalhos relacionados a esta pesquisa e uma comparação entre elas. Desse modo, pode-se analisar os estudos que deram inspiração para este trabalho e assim apresentar as contribuições fornecidas em comparação com outras pesquisas. Portanto, serão apresentadas algumas ontologias que serviram de base para SVNO, assim como, outros trabalhos correlatos.

Dois trabalhos ajudaram a embasar esta pesquisa: o framework esvalue proposto por Gordijn (2002) e a Ontologia de Monitoramento de Valor proposta por de Alencar Silva (2013). Além do mais, o framework esvalue forneceu bases para outras pesquisas, 
como por exemplo o escontrol proposta por Kartseva (2008). O esvalue fornece juntamente com a sua ferramenta, um conjunto de orientações metodológicas, uma notação gráfica, uma ontologia semiformal para o intercâmbio econômico e um mecanismo semiautomatizado de análise de rentabilidade. No entanto, não aborda comportamentos oportunistas em organizações em rede, como a gestão de serviço e aspectos de monitoramento. Portando, a escontrol e a VMO abordam estes dois pontos citados. Enquanto a ezcontrol fornece uma ontologia semiformal como parte de um framework para configurar controles de prevenção do comportamento oportunista em redes de valor; a VMO fornece uma lógica de monitoramento preventivo por meio de uma ontologia formal, para combater o comportamento oportunista na rede. Logo, assim como esta pesquisa, elas também são complementares ao esvalue. [Weigand et al. 2007]. [Normann e Ramirez 1993]

O ezvalue descreve a troca de valor entre os atores de uma rede de valor [Gordijn e Akkermans 2001; Gordijn et al. 2000]. O principal foco é identificar e analisar como o valor é criado, trocado e consumido dentro de uma rede de múltiplos atores [Gordijn e Akkermans 2003]. Esta metodologia é baseada em uma ontologia genérica orientada para o valor especificando o que está em um modelo de e-business. Gordijn e Akkermans (2001), argumentam que é exigido uma articulação do potencial econômico de um modelo de negócios aos analistas de negócios e que o setor não possui técnicas efetivas para expressar e analisar o ponto de vista do valor. Portanto, foi proposta a metodologia esvalue, composta de uma ferramenta e uma ontologia formalizada em UML com algumas restrições OCL.

A Ontologia de Monitoramento de Valor (Value Monitoring Ontology - VMO), foi proposta por de Alencar Silva (2013) como uma ontologia tarefa que descreve uma lógica de monitoramento de serviço para redes de valor. A VMO fornece uma base para o estabelecimento de um ponto de vista de domínio de negócio no monitoramento de serviço. Em vista disso, visa resolver o problema de como uma rede de valor pode ser monitorada. Um dos aspectos mais importantes dessa abordagem é que ela considera o monitoramento como fenômenos comportamentais que ocorrem através das operações realizadas por uma empresa [de Alencar Silva and Weigand 2011a]. Assim, uma rede de valor pode ser monitorada através de uma reconfiguração dos seus papéis de organização interna. Dessa forma, os mesmos atores, atividades de valor e objetos de valor que compõem uma rede de valor normal são reorganizadas como uma rede de valor com monitoramento. De acordo com a VMO, o objeto mais importante de intercâmbio econômico é o objeto de monitoramento. Este tipo de objeto, por sua vez é oferecido em troca de contra-objetos correspondente. De acordo com as diretrizes teóricas fornecidas na VMO, objetos de monitoramento não confiáveis podem desencadear novos problemas de agência sobre as transações de valores que compõem uma rede de valor com monitoramento [de Alencar Silva 2013].

Outro trabalho relacionado e que contribuiu para esta pesquisa é a Enterprise Ontology de Dietz (2006). Contudo, a Enterprise Ontology visa as operações internas de uma empresa. Em vista disso, propõe um conjunto de diretrizes como um modelo ontológico de uma organização. Desse modo, a metodologia tenta extrair apenas a essência de uma organização para elaboração do modelo. Assim como a Enterprise Ontology, a ontologia de modelo de negócios REA (Resource-Event-Agent) proposta por McCarthy (1982) também está focada na empresa. Ela evoluiu a partir de uma estrutura generalizada para modelar sistemas de informações contábeis, para uma ontologia de 
sistemas de informações empresariais [Hruby 2006]. A ontologia é composta de recursos - agentes - eventos e foi formalizada usando diagramas de classes UML.

Analisando a ontologia do esvalue e a SVNO, pode-se identificar semelhanças e diferenças entres estes trabalhos. As semelhanças surgem exatamente pela SVNO ser baseada no esvalue. No entanto, estende o ezvalue, por exemplo, com a inclusão da análise de valores subjetivos no processo de configuração de uma rede de valor. Gordijn (2002) apresenta a importância da sustentabilidade econômica, social e ambiental nas redes de valor. Contudo, aborda apenas a sustentabilidade econômica nos seus modelos de negócios. Neste trabalho, podemos explorar um pouco a sustentabilidade social, ao considerar valores subjetivos, como por exemplo, segurança, privacidade ou confiabilidade, na configuração das redes de valor. Além disso, a SVNO fornece uma lógica de modelagem de redes de valor utilizando artefatos de inteligência artificial.

Para comparar e avaliar a SVNO em relação a estes trabalhos relacionados, será aplicado o framework ONTOMETRIC, proposto por Lozano-Tello e Gómez-Pérez (2004). A ONTOMETRIC é um método que permite aos usuários medir a adequação das ontologias existentes, em relação aos requisitos de seus sistemas. Este método é baseado no processode hierarquia analítica, e pode ser usado para selecionar a ontologia mais adequada entre várias alternativas. Desse modo, o usuário poderá escolher entre a ontologia mais adequada para satisfazer a sua necessidade. Neste trabalho, somente os atributos gerais têm sido considerados. Atributos estes que caracterizam uma ontologia de acordo com seu conteúdo, linguagem de representação, metodologia utilizada, o apoio de ferramentas e custos. Para comparar e avaliar a ontologia proposta neste trabalho com os trabalhos relacionados, será aplicado o método ONTOMETRIC para medir a adequação das ontologias. O método ONTOMETRIC [Lozano-Tello e Gómez-Pérez 2004] permite aos usuários medir a adequação das ontologias existentes, em relação aos requisitos de seus sistemas. Este método é baseado no processo de hierarquia analítica, e pode ser usado para selecionar a ontologia mais adequada entre várias alternativas [Gomez-Perez e Lozano-Tello 2005]. São classificados nas seguintes dimensões: o conteúdo representado na ontologia, a língua em que a ontologia é implementada, a metodologia seguida para desenvolvê-lo, as ferramentas utilizadas para construí-lo, e os custos de utilização da ontologia no sistema. Desenvolvemos as seguintes tarefas para completar o método.

Uma forma de avaliar uma ontologia utilizando o framework ONTOMETRIC [Lozano-Tello e Gómez-Pérez 2004] é analisando o conteúdo representado na ontologia, a linguagem em que a ontologia é implementada, a metodologia seguida para desenvolvêla, as ferramentas utilizadas para construí-la, e os custos de utilização da ontologia no sistema. A partir desses cinco critérios, é possível elaborar uma tabela e fazer uma análise comparativa entre os principais trabalhos relacionados para modelagem de redes de valor. A Tabela 11, apresenta uma análise comparativa baseado no ONTOMETRIC entre o esvalue e a SVNO.

Tabela 11. Analise comparativa baseada no framework ONTOMETRIC entre o esvalue e a SVNO

\begin{tabular}{|c|c|c|}
\hline CRITÉRIOS & $\boldsymbol{E}_{3} \boldsymbol{V A L U E}$ & SVNO \\
\hline Conteúdo & $\begin{array}{c}\text { Design para Modelagem } \\
\text { de Redes de Valor com }\end{array}$ & $\begin{array}{c}\text { Lógica para Modelagem } \\
\text { de Redes de Valor }\end{array}$ \\
\hline
\end{tabular}




\begin{tabular}{|c|c|c|}
\hline & $\begin{array}{c}\text { Suporte a Análise } \\
\text { Econômica da Rede }\end{array}$ & $\begin{array}{c}\text { Semânticas Utilizando } \\
\text { Tecnologias da Web } \\
\text { Semântica com Suporte a } \\
\text { Análise Subjetiva da Rede }\end{array}$ \\
\hline $\begin{array}{c}\text { Linguagem de } \\
\text { especificação }\end{array}$ & $\begin{array}{c}\text { Unified Modeling } \\
\text { Language (UML) }\end{array}$ & $\begin{array}{c}\text { Web Ontology Language } \\
\text { (OWL - DL) }\end{array}$ \\
\hline Metodologia & não identificado & $\begin{array}{c}\text { OTKM (SURE; STAAB; } \\
\text { STUDER, 2009) }\end{array}$ \\
\hline Suporte a ferramenta & $\begin{array}{c}\text { Framework de modelagem } \\
\text { esvalue }\end{array}$ & $\begin{array}{c}\text { Suporta o esvalue e } \\
\text { apresenta um framework } \\
\text { de implementação }\end{array}$ \\
\hline Custo de implementação & $\begin{array}{c}\text { Metodologia de } \\
\text { Modelagem }\end{array}$ & $\begin{array}{c}\text { Raciocínio Utilizando } \\
\text { Motor de Inferência }\end{array}$ \\
\hline
\end{tabular}

Analisando a Tabela 11 pode-se identificar que este trabalho visa apresentar um modelo semântico para modelagem de redes de valor. Em contrapartida, tem-se o esvalue que também pode ser utilizado para esta finalidade. Portanto, os itens a seguir apresentam uma discussão sobre estes dois trabalhos.

- O esvalue é o trabalho relacionado que mais se aproxima desta abordagem. Segundo o critério de conteúdo, o esvalue apresenta um design para modelagem de redes de valor e com suporte a análise econômica da rede, no entanto, ele utiliza uma ontologia semiformal para representar o modelo. Este trabalho permite um upgrade ao esvalue, adicionando uma lógica para modelagem de redes de valor semânticas utilizando tecnologias da web semântica, além de permitir a análise subjetiva da rede.

- A SVNO é especificada utilizando a linguagem OWL-DL, enquanto que o e3value utiliza a Linguagem de Modelagem Unificada (Unified Modeling Language UML). Algumas das vantagens ao utilizar OWL são as correções automáticas e verificações de consistência dos seus axiomas.

- De acordo com a metodologia utilizada, a SVNO apresenta a metodologia de engenharia de ontologias OTKM proposta por Sure, Staab e Studer (2009) no processo de construção da SVNO. Já o esvalue não apresenta nenhuma metodologia especifica no processo de construção do seu modelo.

- O e3value apresenta uma ferramenta desktop com suporte limitado para modelar redes de valor. Este trabalho possui suporte a ferramenta do esvalue, no entanto, apresenta um framework de implementação de um sistema web para modelagem de redes de valor. Esse sistema poderá utilizar-se do processador semântico para inferência de conhecimento na rede.

- O uso de ontologias formais como base de sistemas proporciona uma economia de custos e tempo para reutilização de software. Além do mais, facilita o compartilhamento de informações das redes de valor. O raciocínio baseado na SVNO é realizado utilizando motor de inferência para corretude e consistência da rede, no entanto, o esvalue necessita de interpretação humana e segue seu raciocínio baseado na metodologia de modelagem. 
Desse modo, pode-se perceber que a SVNO é mais apropriada para modelagem de redes de valor semânticas em comparação ao esvalue. No entanto, o usuário pode definir a partir desta análise comparativa a ferramenta mais adequada para resolver o problema.

\section{Conclusão e Trabalhos Futuros}

Neste artigo, foi descrita uma ontologia de tarefa para representação semântica de redes de valor. A ontologia define uma necessidade de negócio como uma composição de um objeto de valor desejado (isto é, uma categoria de produto ou serviço) e seus componentes de valor (isto é, valores objetivos ou subjetivos). A ontologia inclui ainda cinco padrões organizacionais para monitoramento de relações de agência [de Alencar Silva et al. 2017] e um padrão resultante da pesquisa de ação técnica. Esses padrões indicam a proveniência das proposições de valor que poderiam satisfazer a necessidade de negócio de um consumidor. Um conjunto de regras SWRL complementa a ontologia na classificação semiautomática de padrões e seleção de proposições de valor com base na necessidade de criação de valor positivo para o consumidor.

A execução deste projeto de pesquisa foi baseada em diretrizes de Design Science [Wieringa 2014], enquanto que a metodologia de engenharia de ontologias adotada foi aquela proposta por [Sure et al. 2009], por ser uma metodologia indicada para o desenvolvimento de ontologias empresariais. Além disso, a metodologia possui ciclos de refinamento e evolução, os quais podem ser aplicados em múltiplos estudos de casos observacionais.

O processo de validação de ontologia adotado foi aquele proposto por GómezPérez (2004), o qual inclui tarefas de: (1) verificação de corretude, consistência e completude; (2) validação teórica, tecnológica ou pragmática; e (3) avaliação de aceitação, utilidade e usabilidade. A correção e a consistência da ontologia foram verificadas com OWL2 e um plugin SRWL para Protégé [Musen 2015]. A integridade foi verificada de acordo com um Documento de Especificação de Requisitos de Ontologia (ORSD). Para validação, foram usados estudos de caso observacionais na área de música digital [Gordijn e Akkermans 2003], balanceamento de carga em energia renovável [de Alencar Silva e Weigand 2011] e controle aduaneiro [bukhsh e Weigand 2013]. Até agora, a ontologia ainda não foi aplicada em estudos de caso intervencionais ou submetidos à avaliação de analistas de negócios, o que representa uma limitação atual de sua validade. Além do mais, as visualizações em grafos apresentadas são utilizadas apenas para representar as relações entre os conceitos instanciados na ontologia extraidos por meio de consultas SPARQL, não caracterizando a visualização final do sistema.

A principal contribuição apresentada neste trabalho é uma ontologia formal para modelagem de redes de valor semânticas composta por regras, políticas e padrões de consultas. Essa ontologia permite instanciar todos os elementos de uma rede de valor em um modelo formal, possibilitando assim o suporte à verificação automática de modelo, consulta e raciocínio sobre os elementos da rede. Uma segunda contribuição é a lógica de avaliação subjetiva de valores subjetivos, e a sua importância no processo de decisão do consumidor. Outra contribuição é a formalização em OWL-DL dos padrões de monitoramento de agência para configuração de redes de valor. Além das contribuições, uma limitação identificada na ontologia de redes de valor é a necessidade de um padrão de modelagem ou de melhores práticas, a fim de permitir a instanciação dos elementos na 
ontologia. Esta limitação ocorre justamente pelos conceitos da ontologia serem mutuamente definidos, um exemplo é a necessidade de todos os atores realizarem no mínimo uma atividade de valor, já que a comunicação entre o ator e os objetos de valor dar-se-á via atividade de valor. O foco do trabalho ficou concentrado na verificação da utilidade em utilizar uma ontologia formal na modelagem de redes de valor, outra limitação está na usabilidade, já que ainda não apresenta uma interface gráfica simples para manipulação do artefato.

Esta pesquisa compreende três direções de investigação futura. A primeira inclui o desenvolvimento de um Sistema de Apoio à Decisão (SAD) baseado na ontologia. A segunda demanda o uso de estudos de caso intervencionais com Pesquisa-Ação Técnica para validação de requisitos práticos de um grupo de analistas de negócios. A terceira prevê a construção de indicadores de desempenho para avaliação qualitativa de modelos de redes de valor.

\section{References}

Biggemann, S. and Buttle, F. (2012) "Intrinsic value of business-to-business relationships: An empirical taxonomy, Journal of Business Research 65 (8) 11321138 .

Boulic, R. and Renault, O. (1991) "3D Hierarchies for Animation”, In: New Trends in Animation and Visualization, Edited by Nadia Magnenat-Thalmann and Daniel Thalmann, John Wiley \& Sons ltd., England.

Bukhsh, F. A. and Weigand, H. (2011) "Evaluating the application of service-oriented auditing in the B2G domain: A case study", in: Perspectives in Business Informatics Research - 10th International Conference, BIR 2011, Riga, Latvia, October 6-8, 2011. Proceedings, pp. 281-295. doi:10.1007/978-3-642-24511-4_22. URL https://doi.org/10.1007/978-3-642-24511-4_22.

Cameron, K. (1980) “Critical questions in assessing organizational effectiveness, Organizational dynamics" 9 (2) 66-80.

Dyer, S., Martin, J. and Zulauf, J. (1995) "Motion Capture White Paper", ftp://ftp.sgi.com/sgi/A\%7CW/jam/mocap/MoCapWP_v2.0.html, December.

Dietz, J. L. (2006) Enterprise ontology: theory and methodology, Springer Science \& Business Media.

da Silva Reis, J., de Alencar Silva, P., Bukhsh, F. A. and de Castro, A. F. (2018) "Configuring value networks based on subjective business values", in: International Workshop on Value Modeling and Business Ontologies, Springer, 2018.

de Alencar Silva, P., Bukhsh, F. A., da Silva Reis, J., \& de Castro, A. F. (2017). "Agency monitoring patterns for value networks", In International Conference on the Economics of Grids, Clouds, Systems, and Services (pp. 81-93). Springer, Cham.

de Alencar Silva, P. and Weigand, H. (2011) "Enterprise monitoring ontology", in: M. Jeusfeld, L. Delcambre, T.-W. Ling (Eds.), Conceptual Modeling - ER 2011, Springer Berlin Heidelberg, Berlin, Heidelberg, 2011, pp. 132-146.

de Alencar Silva, P., (2013) Value activity monitoring, Tech. Rep. PhD Thesis, Tilburg University. 
European commission: Country fiches for electricity smart metering (accompanying the document), report from the commission, benchmarking smart metering deployment in the eu-27 with a focus on elec-tricity. swd (2014) 188 final, Brussels, 17 june.

Eisenhardt, K. M. (1989) "Agency theory: An assessment and review, Academy of management review" 14 (1) 57-74.

Ferraiolo, D. F. Sandhu, R. Gavrila, S. Kuhn, D. R. Chandramouli, R. (2001) "Proposed nist standard for role-based access control", ACM Transactions on Information and System Security (TISSEC) 4 (3) 224-274.

Gómez-Pérez, A. (2004) “Ontology Evaluation", Springer Berlin Heidelberg, Berlin, Heidelberg, 2004, pp. 251-273. doi:10.1007/978-3-540-24750-0_13. URL https://doi.org/10.1007/978-3-540-24750-0_13

Gomez-Perez, A. Fernández-López, M. and Corcho, O. (2006) “Ontological Engineering: with examples from the areas of Knowledge Management, e-Commerce and the Semantic Web". [S.1.]: Springer Science \& Business Media.

Gomez-Perez, A. and Lozano-Tello, A. (2005) "Applying the ontometric method to measure the suitability of ontologies”. In: Business Systems Analysis with Ontologies. [S.1.]: IGI Global. p. 249-269.

Gordijn, J. and Akkermans, J. (2014) "Value webs, understanding e-business innovation, self-published.

Gordijn, J. P. Leenheer, D. Razo-Zapata, I. (2011) “Generating service value webs by hierarchical configuration: A case in intellectual property rights clearing", in: 2011 44th Hawaii International Conference on System Sciences, 2011, pp. 1-10. doi:10.1109/HICSS.2011.212.

Gordijn, J., and Akkermans, H. (2007) "Business models for distributed generation in a liberalized market environment", Electric Power Systems Research 77 (9) 1178-1188.

Gordijn, J. and Akkermans, J. (2003) "Value-based requirements engineering: exploring innovative e-commerce ideas", Requirements engineering 8 (2) 114-134.

Gordijn, J. (2002) "Value-based Requirements Engineering: Exploring Innovative ecommerce Ideas", PhD Thesis, University Amsterdam.

Gruber, T. R. and Olsen, G. R. (1994) “An ontology for engineering mathematics., KR 94 (1994) 258-269.

Grüninger, M. and Fox, M. S. (1995) "Methodology for the design and evaluation of ontologies". In: Proceedings of IJCAI 1995, Workshop on Basic Ontological Issues in Knowledge Sharing.

Hobbs, J. R. and Pan, F. (2006) “Time ontology in owl”, W3C working draft 27133.

Horridge, M. Drummond, N. Goodwin, J. Rector, A. Stevens, R. Wang, H. (2006) "The manchester owl syntax", in: OWLED 2006 Second Workshop on OWL. Experiences and Directions, Athens, GA, USA.

Horrocks, I., Patel-Schneider, P. F., Boley, H., Tabet, S., Grosof, B., Dean, M. (2004) "Swrl: A semantic web rule language combining owl and ruleml", W3C Member submission 21 (2004) 79. 
Hruby, P. (2006) "Model-driven design using business patterns". [S.1.]: Springer Science $\&$ Business Media.

Kartseva, V. Designing controls for network organizations. [S.1.]: Rozenberg Publishers, 2008.

Lapierre, J. (2000) "Customer-perceived value in industrial contexts", Journal of business \& industrial marketing 15 (2/3) (2000) 122-145.

Lozano-Tello, A. and Gómez-Pérez, A. (2004) "Ontometric: A method to choose the appropriate ontology". Journal of database management, IDEA GROUP PUBLISHING, v. 2, n. 15, p. 1-18.

Mccarthy, W. E. (1982) "The rea accounting model: A generalized framework for accounting systems in a shared data environment". Accounting Review, JSTOR, p. $554-578$.

Morais, E. A. M.; Ambrósio, A. P. L. (2007) "Ontologias: conceitos, usos, tipos, metodologias, ferramentas e linguagens." Relatório Técnico-RT-INF-001/07.

Musen, M. A. (2015) “The protégé project: a look back and a look forward”. AI matters, ACM, v. 1, n. 4, p. 4-12.

Normann, R. and Ramirez, R. (1993) "From value chain to value constellation: Designing interactive strategy.", Harvard business review 71 (4) 65-77.

Noy, N. F. and Mcguinness, D. L. (2001) "Ontology development 101: A guide to creating your first ontology". [S.1.]: Stanford knowledge systems laboratory technical report KSL-01-05 and Stanford medical informatics technical report SMI-2001-0880, Stanford, CA.

O'Sullivan, J. J. (2006) “Towards a precise understanding of service properties", Ph.D. thesis, Queensland University of Technology (2006).

Parasuraman, A., Zeithaml, V. A. and Berry, L. L. (1988) "Servqual: A multiple-item scale for measuring consumer perceived", Journal of retailing 64 (1) 12.

Steedman, I. (1975) "Positive profits with negative surplus value", The Economic Journal 85 (337) (1975) 114-123.

Sure, Y. Staab, S. Studer, R. (2009) "Ontology engineering methodology”, in: Handbook on ontologies, Springer, pp. 135-152.

Searle, J. R. (1969). "Speech acts: An essay in the philosophy of language", Vol. 626, Cambridge university press.

Searle, J. R., Vanderveken, D. (1985) “Foundations of illocutionary logic”, CUP Archive.

Sommerville, I. (2011) "Engenharia de Software”. [S.1.]: Pearson; 9 edition.

Union, E. (2009) "Directive 2009/72/ec of the european parliament and of the council of 13 july 2009 concerning common rules for the internal market in electricity and repealing directive 2003/54/ec, Off. J. Eur. Union L 211 55-93.

Suárez-Figueroa, M. C. Gómez-Pérez, A. and Villazón-Terrazas, B. "How to write and use the ontology requirements specification document". In: Meersman, R.; Dillon, T.; Herrero, P. (Ed.). On the Move to Meaningful Internet Systems: OTM 2009. Berlin, Heidelberg: Springer Berlin Heidelberg, 2009. p. 966-982. ISBN 978-3-642-05151-7. 
Vargo, S. L. and Akaka, M. A. (2009) "Service-dominant logic as a foundation for servisse science: clarifications", Service Science 1 (1) (2009) 32-41.

Vrandečić, D. (2009) “Ontology Evaluation”, Springer Berlin Heidelberg, Berlin, Heidelberg, pp. 293-313. doi:10.1007/978-3-540-92673-3_13. URL https://doi.org/10.1007/978-3-540-92673-3_13

Weigand, H., Johannesson, P., Andersson, B., Bergholtz, M., Edirisuriya, A., Ilayperuma, T. (2007) "Strategic analysis using value modeling-the c3-value approach", in: System Sciences, 2007. HICSS 2007. 40th Annual Hawaii Inter-national Conference on, IEEE, pp. 175c-175c.

Wieringa, R. J. (2014a). "Design Science Methodology for Information Systems and Software Engineering", Springer, Heidelberg.

Wieringa, R. J. (2014b) "Observational case studies”, in: Design Science Methodology for Information Systems and Software Engineering, Springer, 2014, pp. 225-245.

Zeithaml, V. A. (1988) "Consumer perceptions of price, quality, and value: a means-end model and synthesis of evidence, The Journal of marketing 2-22. 